\title{
gु \\ Compensation of the long-range beam-beam interactions as a path towards new configurations for the high luminosity LHC
}

\author{
Stéphane Fartoukh, ${ }^{1, *}$ Alexander Valishev, ${ }^{2, \dagger}$ Yannis Papaphilippou, ${ }^{1}$ and Dmitry Shatilov ${ }^{3}$ \\ ${ }^{1}$ CERN, CH 1211 Geneva 23, Switzerland \\ ${ }^{2}$ Fermi National Accelerator Laboratory, P.O. Box 500, Batavia, Illinois 60510, USA \\ ${ }^{3}$ Budker Institute of Nuclear Physics, SB RAS, Novosibirsk 630090, Russia
}

(Received 3 September 2015; published 1 December 2015)

\begin{abstract}
Colliding bunch trains in a circular collider demands a certain crossing angle in order to separate the two beams transversely after the collision. The magnitude of this crossing angle is a complicated function of the bunch charge, the number of long-range beam-beam interactions, of $\beta^{*}$ and type of optics (flat or round), and possible compensation or additive effects between several low- $\beta$ insertions in the ring depending on the orientation of the crossing plane at each interaction point. About 15 years ago, the use of current bearing wires was proposed at CERN in order to mitigate the long-range beam-beam effects [J. P. Koutchouk, CERN Report No. LHC-Project-Note 223, 2000], therefore offering the possibility to minimize the crossing angle with all the beneficial effects this might have: on the luminosity performance by reducing the need for crab-cavities or lowering their voltage, on the required aperture of the final focus magnets, on the strength of the orbit corrector involved in the crossing bumps, and finally on the heat load and radiation dose deposited in the final focus quadrupoles. In this paper, a semianalytical approach is developed for the compensation of the long-range beam-beam interactions with current wires. This reveals the possibility of achieving optimal correction through a careful adjustment of the aspect ratio of the $\beta$ functions at the wire position. We consider the baseline luminosity upgrade plan of the Large Hadron Collider (HL-LHC project), and compare it to alternative scenarios, or so-called "configurations," where modifications are applied to optics, crossing angle, or orientation of the crossing plane in the two low- $\beta$ insertions of the ring. For all these configurations, the beneficial impact of beam-beam compensation devices is then demonstrated on the tune footprint, the dynamical aperture, and/or the frequency map analysis of the nonlinear beam dynamics as the main figures of merit.
\end{abstract}

DOI: 10.1103/PhysRevSTAB.18.121001

PACS numbers: 29.27.-a, 41.85.-p

\section{INTRODUCTION AND MOTIVATIONS}

Considering a low- $\beta$ insertion (IR) where bunch trains are colliding at the center of the detector, a nonzero crossing angle $\Theta_{c}$ shall be imposed at the interaction point (IP) in order to separate the two beams after the collision. When $\beta^{*}$ is reduced to push the performance of the collider, this angle shall be increased in order to guarantee a sufficiently large normalized beam-beam separation.

For round optics and assuming two low- $\beta$ insertions with horizontal and vertical alternated crossing angle, this separation shall be typically of the order of $10 \sigma$ for the existing layout and collision optics of the Large Hadron Collider (LHC) [1]. For the high-luminosity LHC (HL-LHC) [2], the beam-beam separation will

\footnotetext{
*stephane.fartoukh@cern.ch

†valishev@fnal.gov
}

Published by the American Physical Society under the terms of the Creative Commons Attribution 3.0 License. Further distribution of this work must maintain attribution to the author(s) and the published article's title, journal citation, and DOI. probably need to be increased up to $12.5 \sigma$ [3], due to the larger bunch charge, and longer triplets resulting in the lengthening of the region where the two beams share the same vacuum beam pipe and continue to interact with each other:

$$
\Theta_{c}=\Theta_{c}^{\mathrm{norm}} \times \sqrt{\frac{\epsilon}{\beta^{*}}}=\frac{\Theta_{c}^{\mathrm{norm}} \sigma^{*}}{\beta^{*}},
$$

where $\epsilon$ and $\sigma^{*}=\sqrt{\epsilon \beta^{*}}$ denote the $1 \sigma$ physical beam emittance and the rms spot size at the IP, respectively, and $\Theta_{c}^{\text {norm }}=10-12.5$ refers to the crossing angle normalized by the natural beam divergence at the IP. Increasing the crossing angle, however, affects directly the so-called Piwinski angle $\phi_{w}$, which characterizes the overlap of the two colliding beam distributions, and therefore the luminosity in the presence of a nonzero crossing angle:

$$
\phi_{w} \equiv \frac{\Theta_{c} \sigma_{z}}{2 \sigma^{*}}=\frac{\beta_{w}^{*}}{\beta^{*}}
$$


where $\sigma_{z}$ is the rms bunch length and $\beta_{w}^{*}$ is a characteristic $\beta^{*}$ defined by

$$
\beta_{w}^{*} \equiv \frac{\Theta_{c}^{\text {norm }} \sigma_{z}}{2} .
$$

The potential gain of luminosity with $1 / \beta^{*}$ saturates rapidly below this characteristic $\beta^{*}$, of the order of $\beta_{w}^{*} \sim$ $40-50 \mathrm{~cm}$ for typical LHC or HL-LHC parameters:

$$
\mathcal{L}\left(\beta^{*}\right) \propto \frac{1}{\beta^{*} \sqrt{1+\phi_{w}^{2}}}=\frac{1}{\beta^{*} \sqrt{1+\left(\beta_{w}^{*} / \beta^{*}\right)^{2}}} \stackrel{\beta^{*} \ll \beta_{w}^{*}}{\longrightarrow} \frac{1}{\beta_{w}^{*}} .
$$

A potential mitigation measure is offered by the use of flat optics, with a $\beta^{*}$ as small as possible in the parallel separation plane, down to $\beta_{\|}^{*} \sim \sigma_{z}$, and of the order of $\beta_{\mathrm{X}}^{*} \sim \beta_{w}^{*}$ in the crossing plane [4]. Indeed, this strategy still leads to an increase of the luminosity with $1 / \sqrt{\beta_{\|}^{*}}$, until it saturates again due to the hourglass effect when $\beta_{\|}^{*}$ becomes comparable to the rms bunch length. For flat optics, however, even an alternated horizontal and vertical crossing angle in the two low- $\beta$ insertions of the ring does no longer warrant the full self-compensation of the tune shift and, in general, of the $4 n+2$-pole like tune spread induced by the long-range (LR) beam-beam interactions (see Sec. II B for an analytical treatment). As a result, the normalized crossing angle shall be increased beyond the canonical values mentioned above for round optics, and substantially for large $\beta^{*}$ aspect ratio. The typical rule of thumb for the (HL-) LHC is that a flat optics with a $\beta^{*}$ aspect ratio of $r^{*}=$ $\beta_{\mathrm{X}}^{*} / \beta_{\|}^{*} \sim 4$ requires almost the same crossing angle in $\mu \mathrm{rad}$ (and the same magnet aperture) as a round optics with an equivalent $\beta^{*}$ of $\beta_{\text {round }}^{*} \equiv \sqrt{\beta_{\mathrm{X}}^{*} \beta_{\|}^{*}}[3,5]$ :

$$
\begin{aligned}
\Theta_{c, \text { flat }} & \sim \Theta_{c, \text { round }} \quad \text { when } \beta_{\mathrm{X}}^{*}=2 \beta_{\text {round }}^{*} \\
\text { and } \quad \beta_{\|}^{*} & =\beta_{\text {round }}^{*} / 2 .
\end{aligned}
$$

This corresponds to an increase of the normalized crossing angle by about $\sqrt{2}$ in the case of flat optics with a $\beta^{*}$ aspect ratio of 4 , compared to a round optics of equivalent $\beta^{*}$ :

$$
\begin{aligned}
\Theta_{c, \text { flat }}^{\text {norm }} & \equiv \Theta_{c, \text { flat }} / \sqrt{\frac{\epsilon}{\beta_{\mathrm{X}}^{*}}} \sim \Theta_{c, \text { round }} / \sqrt{\frac{\epsilon}{2 \beta_{\text {round }}^{*}}} \\
& =\sqrt{2} \times \Theta_{c, \text { round }}^{\text {norm }} .
\end{aligned}
$$

Therefore the Piwinsky angle is only reduced by a factor $\sqrt{2}$ with respect to an equivalent round optics, instead of by a factor of 2 if the normalized crossing angle could be kept at around $10-12.5 \sigma$ for flat optics as well. Consequently, finding means to keep the normalized crossing angle as low as possible when flattening the optics, for example through the use of electromagnetic wires proposed in [6], or similarly electron lenses [7,8] [see sketch in Fig. 1(b)], would offer a very interesting path toward a powerful performance upgrade. This possibility, the so-called HL-LHC Plan B, was effectively introduced in the project [9] and fully simulated very recently [10].

The baseline scenario (Plan A) relies on a different "head-on collision device," the so-called crab cavities. Crab cavities are deflecting rf dipoles [11,12] which aim at maximizing the overlap of the two beam distributions despite of the crossing angle [see Fig. 1(a)], in order to fully restore the luminosity gain with $1 / \beta^{*}$. The HL-LHC project has chosen this second option as a baseline, despite a challenging hardware implementation, since it offers in principle better luminosity performance. Nevertheless, even in this case, a reduction of the crossing angle would still be beneficial for (i) the crab-cavity voltage needed and the strength of the orbit corrector magnets which are involved in the crossing bumps, (ii) the mechanical aperture needed by the beam, and/or (iii) the heat load and integrated radiation dose coming from the debris produced at the IP and deposited in the final focus quadrupoles.

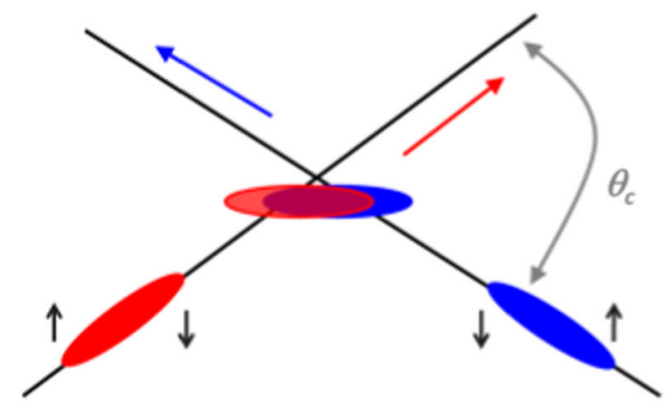

(a)

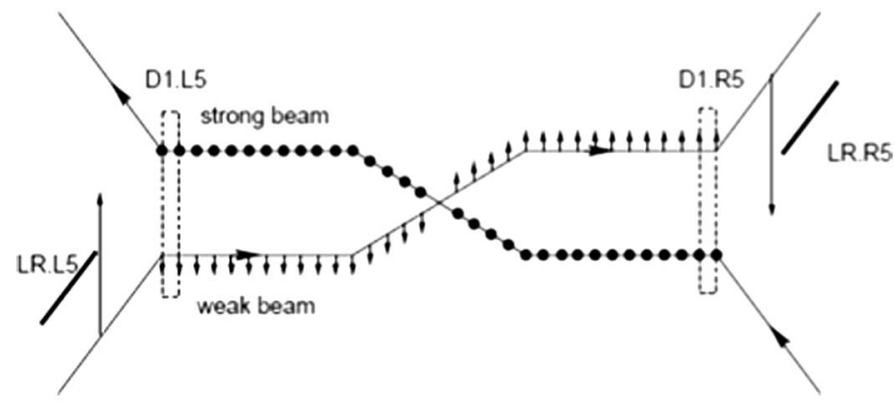

(b)

FIG. 1. Possible "head-on collision tools" to maximize the overlap of the two beam distributions at the interaction point, and therefore maximize the machine performance in the presence of a nonzero crossing angle: bunch rotation induced by crab-cavities at constant crossing angle (left picture, courtesy of F. Zimmerman), current bearing wires (or electron lens) to compensate for the long-range (LR) beam-beam effects and reduce the crossing angle itself (right picture [6], courtesy of J. P. Koutchouk), or any combination of the two. 
As already mentioned, the idea of using a current bearing wire to compensate for the LR beam-beam interactions was introduced in [6]. Then several beam experiments were carried out installing and testing this device in different machines [13-16], with in particular very clear beneficial effects observed on the beam lifetime for the $e^{+} e^{-} \mathrm{DA} \Phi \mathrm{NE}$ collider. On the other hand, to the best knowledge of the authors, the azimuthal positioning of this device in the collider (and the corresponding aspect ratio of the $\beta$ functions) and its settings (in terms of integrated current and transverse distance with respect to the circulating beam) have always been optimized iteratively (see e.g., [17]) and more or less empirically. This is where a more systematic and deterministic approach would be appropriate in view of the huge potential of this device.

Using the weak-strong beam-beam approximation, the next section develops a semianalytical method for the compensation of the LR beam-beam interactions assuming two current wires acting on the weak beam and located symmetrically on either side of the IP [see Fig. 1(b)]. In the particular case of the (HL-)LHC, it will be shown that a very clean correction can be achieved provided these two wires are located at a position where the $\beta$ aspect ratio $r \equiv \beta_{x} / \beta_{y}$ is close to either 2 or $1 / 2$, i.e., right after the separation dipole D1 or in between Q4 and Q5 for typical antisymmetric (HL-)LHC collision optics. Using the beambeam tune footprint, the dynamic aperture and/or the frequency map analysis as figures of merit, Sec. III will then illustrate the potential benefit of this device in many different machine configurations, i.e., not only for the HL-LHC baseline with round optics and a vertical and horizontal (HV) alternated crossing angle of about $600 \mu \mathrm{rad}$ in IR1 and IR5, but also for alternative scenarios with a substantially smaller crossing angle in round or flat optics mode, or assuming an $\mathrm{HH}$ or VV orientation of the crossing planes in the two high luminosity insertions of the ring.

\section{CORRECTION ALGORITHM}

\section{A. Multipole expansion of the long-range beam-beam interaction}

Let us consider two slices of charged particles traveling at the speed of light in opposite (nearly) parallel directions, and interacting with each other at time $t=0$ and abscissa $s=s_{0}$. Let us note $Q_{0}$ the total charge of the first slice ("strong" beam), $r_{0}$ the radius characterizing its finite transverse extension, and $z_{0} \equiv z_{1}-z_{2} \equiv x_{0}+i y_{0}$ (in complex notations) the transverse position of the centroid of the first slice with respect to that of the second slice ("weak" beam). A test particle of the weak beam, at a transverse position $z \equiv x+i y$ with respect to its centroid, has a transverse position $z-z_{0}$ relative to the centroid of the strong beam. Taking the centroid of the strong beam as the origin of the transverse plane, orienting the longitudinal $s$ coordinate along the trajectory $t(s)=\left(s-s_{0}\right) / c$ of the test particle, and assuming $\left|z-z_{0}\right| \gg r_{0}$, the electric and magnetic fields seen by this particle are purely radial and azimuthal, respectively, and given by the well-known expressions:

$$
\begin{aligned}
E_{r} & =-c B_{\theta} \\
& =\frac{Q_{0}}{2 \pi \epsilon_{0}\left|z-z_{0}\right|} \times \delta\left(s-s_{0}+c t\right) \\
& =\frac{Q_{0}}{4 \pi \epsilon_{0}\left|z-z_{0}\right|} \times \delta\left(s-s_{0}\right),
\end{aligned}
$$

where $\epsilon_{0}$ is the permittivity of free space, the time $t$ has been substituted by $t=\left(s-s_{0}\right) / c$, and the relation $\delta(2 s) \equiv \delta(s) / 2$ has been used for the Dirac function. The integrated Lorentz force seen by the test particle is therefore radial, corresponding to an effective integrated magnetic field which, written in complex notation, is given by

$$
\begin{aligned}
\int d s\left[B_{y}+i B_{x}\right]_{\mathrm{eq}} & \equiv 2 e^{-i \theta} \int d s B_{\theta} \\
& =-\frac{Q_{0}}{2 \pi \epsilon_{0} c} \times \frac{\cos (\theta)-i \sin (\theta)}{\left|z-z_{0}\right|},
\end{aligned}
$$

with $\theta$ being the argument of $\left(z-z_{0}\right)$. Multiplying the numerator and denominator by $\left|z-z_{0}\right|$, and simplifying by the complex conjugate of $\left(z-z_{0}\right)$ occurring in the numerator, one finally gets

$$
\begin{aligned}
\int d s\left[B_{y}+i B_{x}\right]_{\mathrm{eq}} & =-\frac{\mu_{0}(I L)_{\mathrm{eq}}}{2 \pi} \times \frac{1}{z-z_{0}} \\
\text { with } \quad(I L)_{\mathrm{eq}} & \equiv Q_{0} c,
\end{aligned}
$$

where $\mu_{0}=1 / \epsilon_{0} / c^{2}$ denotes the permeability in free space. One therefore easily recognizes the integrated magnetic field induced by a wire of length $L$ and carrying a current $I$ with $I \times L \equiv Q_{0} c$. Whether the strong beam is an infinitely thin slice or a bunch of charged particles will not change the above result, under the condition that the distance $\left|z-z_{0}\right|$ does not vary along the finite length of the interaction, which is a very good approximation in the case of the (HL-) LHC. Assuming $\left|z / z_{0}\right|<1$ in the above relation (which means that the transverse distribution of the two beams does not overlap at the parasitic encounters), the equivalent integrated magnetic field seen by the test particle can be easily expanded in multipole harmonics:

$$
\begin{aligned}
\int d s\left[B_{y}+i B_{x}\right]_{\mathrm{eq}} & =\sum_{k=1}^{\infty}\left[B_{k}+i A_{k}\right] z^{k-1} \\
\text { with } \quad B_{k}+i A_{k} & \equiv \frac{\mu_{0}(I L)_{\mathrm{eq}}}{2 \pi} \times \frac{1}{z_{0}^{k}}
\end{aligned}
$$




\section{B. Analogy with standard multipole magnets, and effect of multiple interaction regions}

Equation (10) contains a number of important aspects. First of all, for horizontal crossing, i.e., assuming $z_{0}=x_{0}$ to be real in (10), the LR beam-beam interaction only contains normal multipole components $\left(A_{k}=0\right.$ for all $k$ 's). For vertical crossing, i.e., assuming $z_{0}=i y_{0}$ to be purely imaginary, the odd harmonics are skewed $\left(B_{2 k+1}=0\right)$, while the even harmonics are normal $\left(A_{2 k}=0\right)$. Furthermore, assuming two insertions with strictly equivalent optics and the same normalized crossing angle, but the crossing plane rotated by $\pm \pi / 2$ when passing from the first IP to the second one, i.e., $z_{0_{\mathrm{IP} 2}} \equiv \pm i z_{0_{\mathrm{IPP}}}$, then the tune shift and tune spread induced by the $4 n+2$ harmonics ( $B_{2}$ for the tune shift, and $B_{6}, B_{10}, \ldots$ for the tune spread) selfcompensate between the two insertions, while the $4 n$-pole like detuning terms add up. The possible utilization of crosstalks between crossing angle orientation and tune shift and tune spread induced by the LR beam-beam interactions emerged in the early design phase of the Superconducting Super Collider (SSC) $[18,19]$. This led to the proposal of an alternated $\mathrm{HV}$ crossing scheme in the two experiments of the SSC machine, which was proposed a decade later for the two high luminosity insertions of the LHC (see e.g., [20]). However, an important consequence of this choice is that the tune spread generated by the LR beam-beam interactions is very close to that induced by a pure octupole, since the next nonzero first order contribution to the tune spread is $B_{8}$-like. As a result this tune spread can be compensated very cleanly by an inappropriate choice of the Landau octupole polarity. This was effectively the case until the very end of the LHC Run I when this effect was realized, and the polarity of the LHC Landau octupole was changed from negative to positive, and now kept for Run II in order to ensure coherent beam stability through the Landau damping at the end of the squeeze [21] (see also [22] for a further analysis of the same effect in terms of Landau stability diagram). Finally, targeting a full self-compensation to first order of all the detuning terms induced by the LR beam-beam interactions, (i.e., not only from the components $B_{2}, B_{6}, \ldots$, but also from $\left.B_{4}, B_{8}, \ldots\right)$, one possible configuration would be a ring equipped with four low- $\beta$ insertions strictly identical in terms of optics ( $\beta$ functions), layout, and normalized beambeam separation, but with a crossing plane rotated by $\pi / 4$ when passing from one insertion to the next one, i.e., $z_{0_{\mathrm{IP} k}} \equiv \pm e^{i(k-1) \pi / 4} z_{0_{\mathrm{IP1}}}, k=1, \ldots, 4$.

\section{Correction method applied to the HL-LHC}

\section{Features of the (HL-)LHC optics}

Let us consider a typical left/right antisymmetric HL-LHC collision optics with the same $\beta^{*}$ in the two transverse planes [round optics as shown in Fig. 2(b)], and two wires installed symmetrically on either side of the IP [see Fig. 1(b)] at an aspect ratio $r_{w}$ of the $\beta$ functions defined by

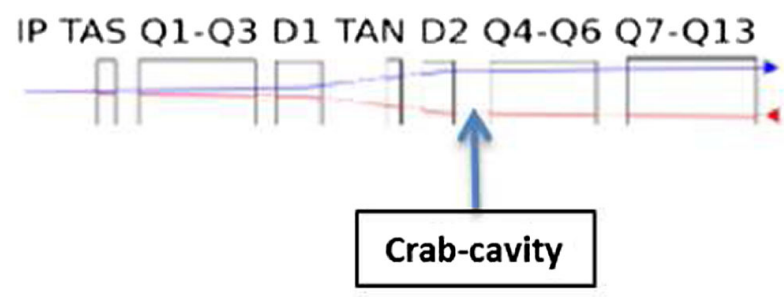

(a)

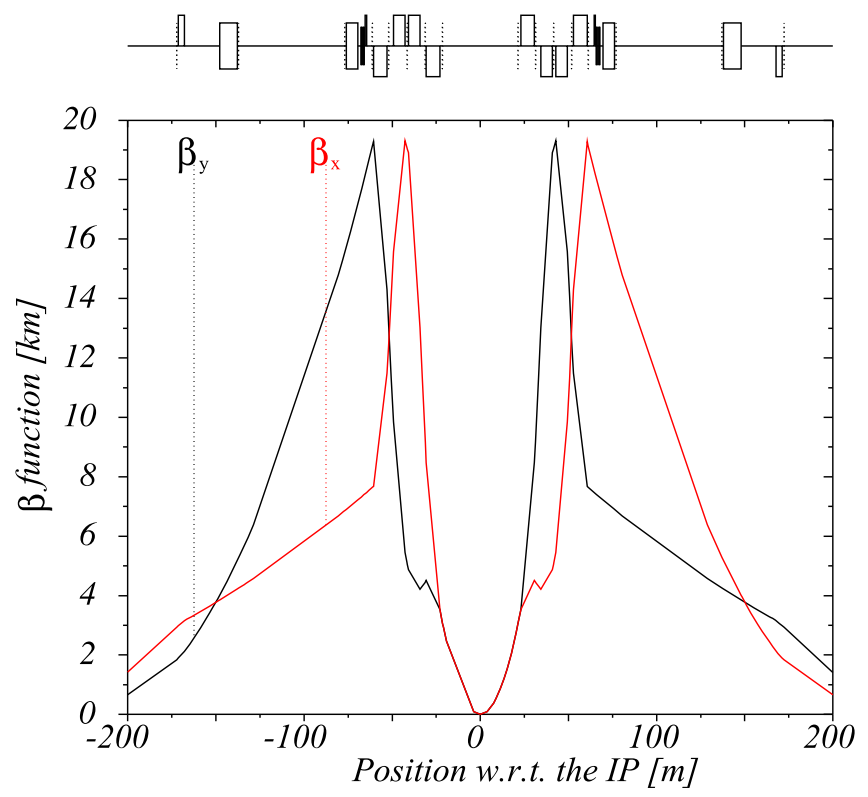

(b)

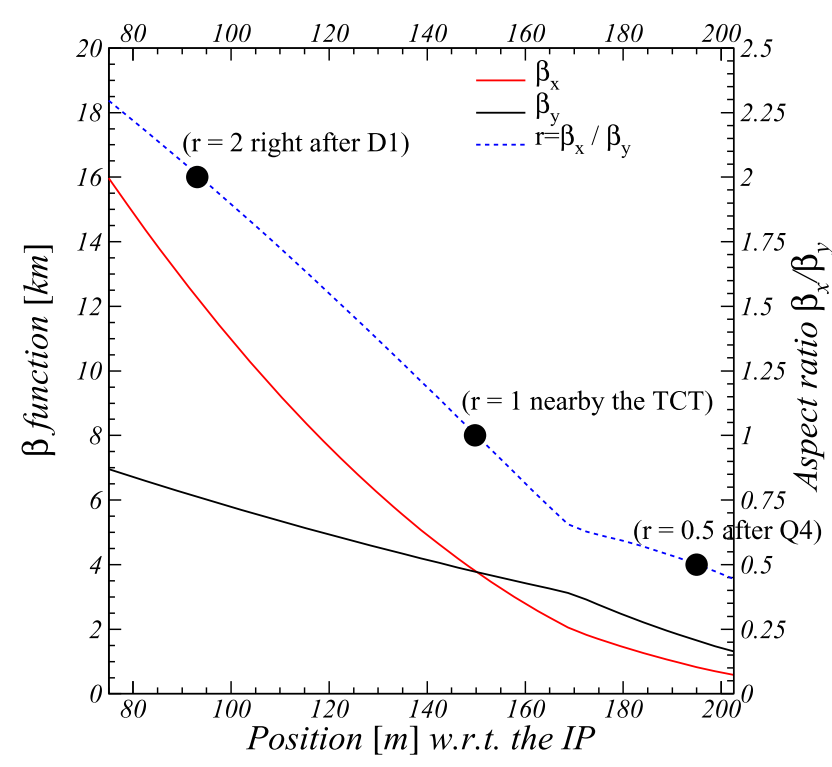

(c)

FIG. 2. Sketch of the layout for the new high luminosity insertions of the HL-LHC (a), typical HL-LHC collision optics with $\beta^{*}=15 \mathrm{~cm}(\mathrm{~b})$, and zoom on the $\beta$ functions and corresponding aspect ratio (c) from the exit of the separation dipole D1 to an intermediate position in between the Q4 and Q5 matching quadrupoles. 


$$
r_{w} \equiv \frac{\beta_{x}^{w} \cdot \mathrm{R}}{\beta_{y}^{w \cdot \mathrm{R}}}=\frac{\beta_{y}^{w . \mathrm{L}}}{\beta_{x}^{w . \mathrm{L}}},
$$

where the second equality is true only for round optics. This aspect ratio is shown in Fig. 2(c) as a function of the distance from the IP for a typical HL-LHC collision optics and the clockwise rotating beam (Beam1), assuming $\beta^{*}=15 \mathrm{~cm}$ in both transverse planes. It is of the order of $r_{w} \sim 2$ right after the separation dipole D1, $r_{w} \sim 1$ close to the recombination dipole D2 and tertiary collimator (TCT), and an aspect ratio of $r_{w} \sim 0.5$ is reached in between the first and second matching quadrupoles Q4 and Q5. However, provided additional constraints are imposed on the inner triplet layout and/or inserted into the optics matching procedure, the longitudinal position where the two $\beta$ functions are the same, i.e., $\left(r_{w} \equiv 1\right)$, can be shifted well in between D1 and $\mathrm{D} 2$, and a $\beta$ aspect ratio of $1 / 2$ can be established much closer to the TCT.

Let us consider $2 \times N_{\mathrm{LR}}$ parasitic beam-beam encounters occurring every $3.75 \mathrm{~m}$ on either side of the IP (for a bunch spacing of $25 \mathrm{~ns}$ ) until the beam enters the D1 separation dipole. In the remainder of this paper this number will be set to $N_{L R}=18$, corresponding to the intermediate layout version SLHCV3.1b [23] of the new HL-LHC insertions with a $150 \mathrm{~T} / \mathrm{m}$ triplet $(140 \mathrm{~mm}$ aperture), compared to $N_{\mathrm{LR}}=15$ for the existing LHC triplet and very likely $N_{\text {LR }}=21$ for the final HL-LHC triplet layout [24] with an operational gradient of $130-132 \mathrm{~T} / \mathrm{m}(150 \mathrm{~mm}$ aperture). For $\beta^{*}=15 \mathrm{~cm}$ with an horizontal crossing angle normalized to $12.5 \sigma$ (i.e., $590 \mu \mathrm{rad}$ at $7 \mathrm{TeV}$ for a normalized emittance of $\gamma \epsilon=2.5 \mu \mathrm{rad}$ ), the beam-beam separation is shown in Fig. 3, given in mm in Fig. 3(a) and normalized by the horizontal beam size of Beam1 in Fig. 3(b). The non-normalized beam-beam separation $d_{b b}$ is quasisymmetric between the left and the right sides of the IP and given by $d_{b b}(s) \sim d_{b b}(-s) \approx\left[\sqrt{\beta_{x}(s) \beta^{*}}+\sqrt{\beta_{y}(s) \beta^{*}}\right] \Theta_{c} / 2$,

both in horizontal and vertical crossing. These symmetries are related to the following features of the LHC optics: first the antisymmetry of the optics for a given beam between the left and right sides of the IP (i.e., $\beta_{x}^{\mathrm{L}}=\beta_{y}^{\mathrm{R}}$ and $\left.\beta_{x}^{\mathrm{R}}=\beta_{y}^{\mathrm{L}}\right)$, but also the antisymmetry of the optics between the two beams on a given side of the IP (i.e., $\beta_{x}^{b 2}=\beta_{y}^{b 1}$ and $\beta_{x}^{b 1}=\beta_{y}^{b 2}$ ), and finally from the fact that most of the crossing angle is generated by orbit correctors located on the non-ip side of the triplet, with a phase advance from the IP to the parasitic encounters, which is quasiconstant and very close to $\pm \pi / 2$ (see Fig. 4).

When normalized by the beam size in the crossing plane [see Fig. 3(b)], these symmetries are broken and the normalized beam-beam separation is given by

$$
d_{b b}^{\text {norm }}(s) \approx\left[1+\sqrt{\beta_{y, x}(s) / \beta_{x, y}(s)}\right] \Theta_{c}^{\text {norm }} / 2,
$$

for horizontal and vertical crossing, respectively, and where the normalized crossing angle $\Theta_{c}^{\text {norm }}$ has been introduced in Eq. (1). In the drift space between the IP and the first final focus quadrupole, the normalized beam-beam separation is therefore constant $\left(\beta_{x}(s)=\beta_{y}(s)=\beta^{*}+s^{2} / \beta^{*}\right)$, but is varying significantly in the inner triplet quadrupoles where the aspect ratio of the $\beta$ functions is no longer constant. As a result, the quality of the correction of the LR beam-beam interactions is expected to be strongly dependent on the aspect ratio $r_{w}$ of the $\beta$ functions at the wire position. Also, in a configuration with only two wires (or e-lenses) per beam and per IP (i.e., only two independent knobs within a multiplicative constant given by the wire current which shall naturally scale with the beam intensity), only two

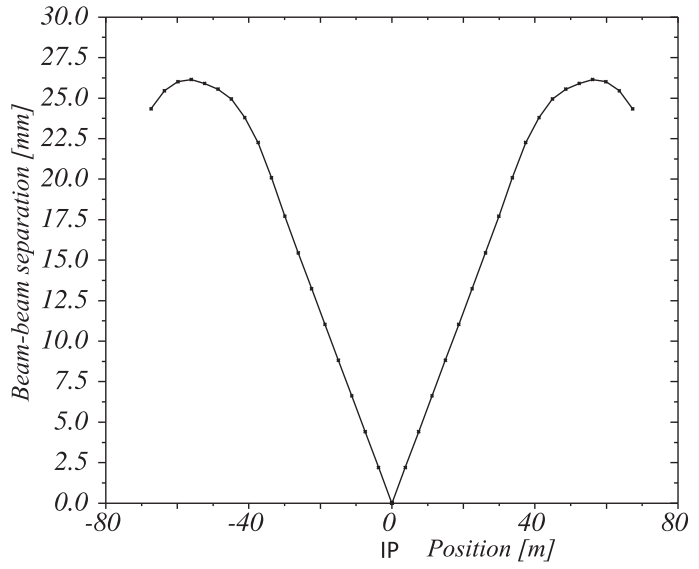

(a)

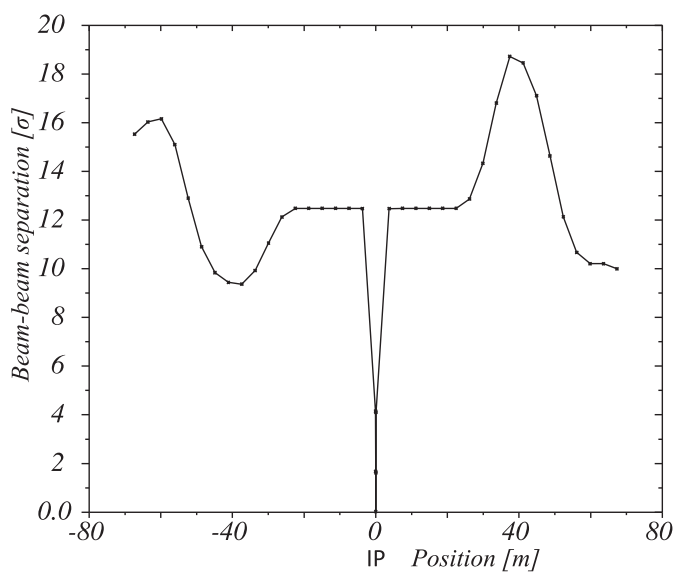

(b)

FIG. 3. Beam-beam separation for a horizontal crossing angle of $590 \mu \mathrm{rad}$. The separation is shown in $\mathrm{mm}$ in (a), and normalized by the horizontal beam size of the clockwise rotating beam in (b) (assuming $\beta^{*}=15 \mathrm{~cm}$, a beam energy of $7 \mathrm{TeV}$ and a normalized emittance of $\gamma \epsilon=2.5 \mu \mathrm{rad}$ ). The markers indicate the positions of the LR beam-beam encounters, except at the IP where the head-on collision is modeled by five beam-beam slices [as clearly visible, assuming no crab-cavity, in (b)]. 


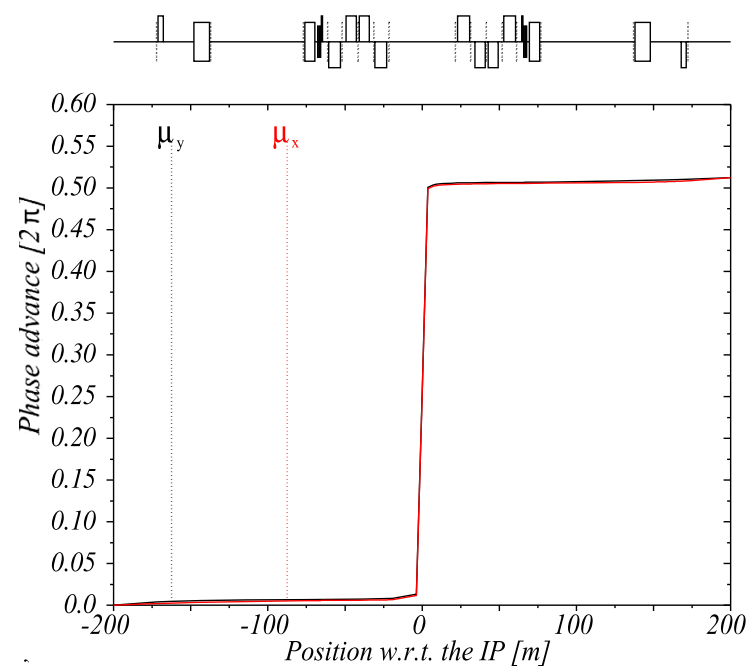

(a)

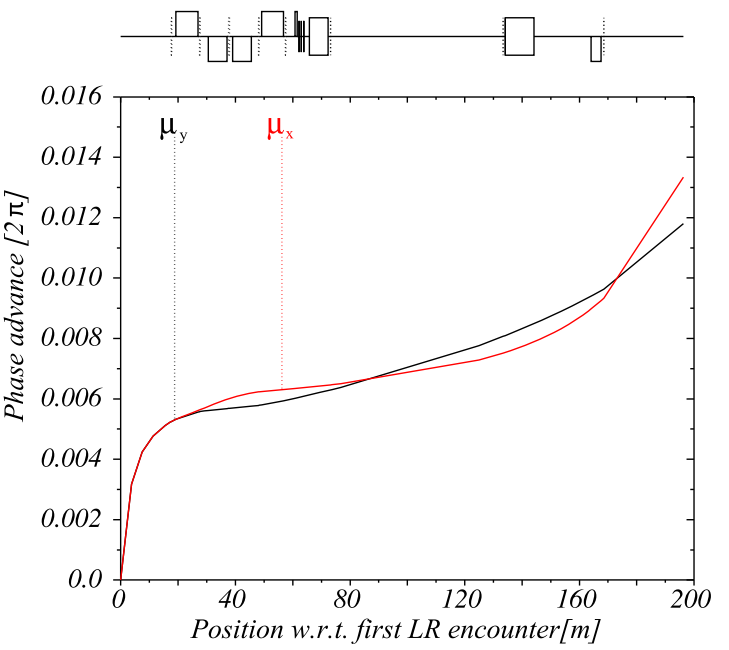

(b)

FIG. 4. Evolution of the betatron phase advances over a distance of $\pm 200 \mathrm{~m}$ on either side of the IP (a), then zoomed on the right side taking the origin at the first parasitic encounter located at $3.75 \mathrm{~m}$ with respect to the IP. When the optics is squeezed $\left(\beta^{*}=15 \mathrm{~cm}\right.$ in this case), the betatron phases jump by $\pi$ across the IP. These phases then stabilize at a value very close to $\pm \pi / 2$ within a few degrees over a relatively long distance of about $200 \mathrm{~m}$.

resonance driving terms (RDTs) or two detuning terms can a priori be compensated simultaneously. Later, one will see however that (i) RDTs and detuning terms of the same order are very close to each other, (ii) due to the antisymmetry of the (HL-)LHC optics not 2 but actually 4 RDTs can be zeroed out simultaneously (provided the left and right wires are positioned symmetrically with respect to the IP), and (iii) all RDTs in fact can be strongly minimized provided an appropriate choice of the aspect ratio of the $\beta$-function at the wire location.

\section{Correction algorithm for round optics}

When the HL-LHC optics is squeezed, the betatron phases jump by $\pi$ in both planes from the left to the right side of the IP. Then, these phases stay quasiconstant, within a few degrees, from the first parasitic encounter, at $\pm 3.75 \mathrm{~m}$ with respect to the IP, until the zone Q4/Q5 located at about $\pm 200 \mathrm{~m}$ from the IP (see Fig. 4). The detuning terms are therefore included in the list of RDTs induced by the LR beam-beam interactions. For instance, the horizontal anharmonicity $\partial Q_{x} / \partial J_{x}$ is simply proportional to the modulus of the $(4,0)$ RDT, since the betatron phase advances are vanishing or close to $\pi$ between the different LR beam-beam encounters of a given interaction region. From the multipole expansion obtained in (10), and restricting our discussion to purely horizontal or vertical crossing, the RDTs to be corrected are then proportional to the following quantities:

$c_{p q}^{\mathrm{LR}} \equiv \sum_{k \in \mathrm{LR}} \frac{\beta_{x}^{p / 2}\left(s_{k}\right) \beta_{y}^{q / 2}\left(s_{k}\right)}{d_{b b}^{p+q}\left(s_{k}\right)}, \quad p \geq 0, \quad q \geq 0$ where the summation is taken over $2 \times N_{\mathrm{LR}}$ parasitic beambeam encounters taking place on either side of the IP, $p$ and $q$ are integers, $q$ is always an even number in the case of $\mathrm{H}$ crossing (leading to normal resonances), and idem for $p$ in the case of $\mathrm{V}$ crossing (leading to normal or skew resonances when $p+q$ is an even or an odd integer, respectively). It is worth noting that the phasor term $(-1)^{p+q}$ related to the $\pi$ phase jump between the left and the right side of the IP is apparently missing in the above relation: it is in fact absorbed in the convention chosen for the beam-beam separation $d_{b b}\left(s_{k}\right)$, taken positive on both sides of the IP. Finally due to the optics antisymmetry, and the $s$-parity of the function $d_{b b}(s)$, the driving terms $c_{p q}$ and $c_{q p}$ are clearly the same (when both are excited, i.e., when both $p$ and $q$ are even integers).

Similar quantities can then be defined for the two wires (or e-lenses) installed on the left (L) and right (R) sides of the IP:

$$
\left\{\begin{array}{l}
c_{p q}^{w \cdot \mathrm{L}} \equiv N_{w \cdot \mathrm{L}} \times \frac{\left(\beta_{x}^{w . \mathrm{L}}\right)^{p / 2}\left(\beta_{y}^{w . \mathrm{L}}\right)^{q / 2}}{\left(d_{w . \mathrm{L}}\right)^{p+q}} \\
c_{p q}^{w \cdot \mathrm{R}} \equiv N_{w \cdot \mathrm{R}} \times \frac{\left(\beta_{x}^{w \cdot \mathrm{R}}\right)^{p / 2}\left(\beta_{y}^{w \cdot \mathrm{R}}\right)^{q / 2}}{\left(d_{w \cdot \mathrm{R}}\right)^{p+q}}
\end{array}\right.
$$

with $N_{w . \mathrm{L} / \mathrm{R}}$ describing the integrated current of the left and right wires, expressed in terms of equivalent number of parasitic beam-beam encounters [see Eq. (9) for the correspondence], and where the quantities $d_{w . \mathrm{L} / \mathrm{R}}$ are positive and denote the transverse positions of the left and right wires with respect to the weak beam to be corrected [with the wires always located in between the strong and weak beam as shown in Fig. 1(b)]. For round optics, the RDTs induced by the left and right wires can also be written as 


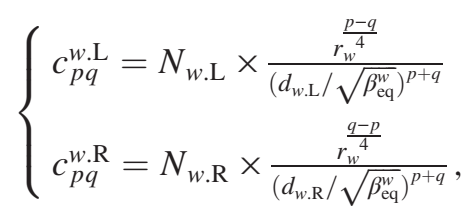

with $\quad \beta_{\mathrm{eq}}^{w} \equiv \sqrt{\beta_{x}^{w \cdot \mathrm{R}} \beta_{y}^{w \cdot \mathrm{R}}}=\sqrt{\beta_{x}^{w \cdot \mathrm{L}} \beta_{y}^{w . \mathrm{L}}}$.
In this case, and avoiding the degenerated cases defined by $r_{w}^{\frac{q-p}{4}} \equiv 1$, where the action of the left and right wires are the same on one specific RDT (when $p \equiv q$ ) or on all RDTs simultaneously (when $r_{w} \equiv 1$ ), requiring that the wires are adjusted to compensate two given RDTs, namely $c_{p_{1} q_{1}}^{\mathrm{LR}}$ and $c_{p_{2} q_{2}}^{\mathrm{LR}}$ with $p_{1} \neq q_{1}$ and $p_{2} \neq q_{2}$, and that their global action features the above mentioned symmetries when exchanging $p$ into $q$, i.e., $c_{p q}^{w . \mathrm{L}}+c_{p q}^{w . \mathrm{R}} \equiv c_{q p}^{w . \mathrm{L}}+c_{q p}^{w . \mathrm{R}} \equiv c_{p q}^{\mathrm{LR}}=c_{q p}^{\mathrm{LR}}$, one gets:

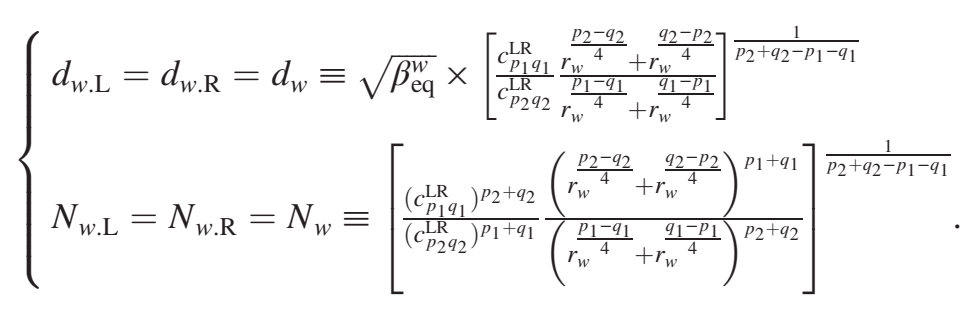

\section{Wire setting variations with $\beta^{*}$ and crossing angle, and generalization to flat optics}

The low- $\beta^{*}$ collision optics of the HL-LHC relies on the deployment of the so-called achromatic telescopic squeezing (ATS) scheme [25], in which case the settings of the triplet and matching quadrupoles are strictly constant below a certain $\beta^{*}$ (of typically $40-50 \mathrm{~cm}$ ), both for round and flat optics. As a result, and due to the quasiconstant betatron phase advances, close to $\pm \pi / 2$ between the IP and any location at a few meters up to $\sim \pm 200 \mathrm{~m}$ upstream or downstream, the $\beta$ functions at the parasitic encounters, namely $\beta_{x, y}\left(s_{k}\right)$, but also at the wires, $\beta_{x, y}^{w . L, w . R}$, scale with the inverse of $\beta_{x, y}^{*}$. On the other hand, the (non-normalized) beam-beam separation $d_{b b}\left(s_{k}\right)$ is simply proportional to the crossing angle $\Theta_{c}$. Consequently, the RDTs induced by the LR beam-beam interactions and the wires possess the following scaling laws:

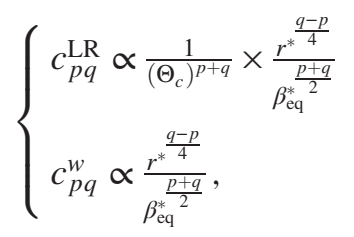

where $r^{*} \equiv \beta_{x}^{*} / \beta_{y}^{*}$ is the aspect ratio of the $\beta$ functions at the IP, and $\beta_{\mathrm{eq}}^{*} \equiv \sqrt{\beta_{x}^{*} \beta_{y}^{*}}$. Since the scaling with $\beta_{x, y}^{*}$ is the same for the coefficients $c_{p q}^{\mathrm{LR}}$ and $c_{p q}^{w}$, adjusting the wires in strength and position following (18) for two selected RDTs, for a given round optics, and a given crossing angle, automatically warrants the cancellation of the same RDTs for any other collision optics of sufficiently small $\beta^{*}$, round or flat, keeping constant the (non-normalized) crossing angle. In this case, of course, the residual of the noncorrected driving terms cannot be kept constant when changing $\beta_{x, y}^{*}$. On the other hand, if one assumes the existence of an optimal aspect ratio $r_{w}$ at the wire position for which all the RDTs could be minimized simultaneously for a given round optics, then the corresponding positioning of the wires in the layout, their strength and transverse position will also be very close to optimal for any other collision optics, round (for which $r_{w}$ will be kept unchanged) or flat (in which case $r_{w}$ will scale with $\left.1 / r^{*}\right)$. Surprisingly, such optimal positions exist for the (HL-)LHC optics, as we will see later on. Finally, when changing the crossing angle, the transverse position of the left and right wires shall be modified in proportion, but their current kept constant:

$$
d_{w} \propto \Theta_{c} \quad \text { and } \quad N_{w}=\text { Cst }
$$

Similarly to the present LHC, the optics squeeze in the HL-LHC is foreseen to be performed at constant physical (non-normalized) crossing angle, without any further change during the luminosity leveling process, in particular for the HL-LHC baseline scenario relying on luminosity levelling with $\beta^{*}$. Therefore, (i) ramping up the wire current toward the end of the ramp, (ii) fixing their transverse positions at flat top energy as a function of the crossing angle established just before the squeeze (when the normalized crossing angle is still very large and therefore both the effects the LR interactions and of the wires are marginal), (iii) keeping these positions strictly constant later on, and (iv) reducing the wire current following the smooth evolution of the beam intensity during the physics coast, offer a very simple and optimal usage of this new device in operation. 


\section{Aspect ratio optimization for the $\beta$ functions at the wire position}

For the HL-LHC baseline collision optics with $\beta_{x, y}^{*}=$ $15 \mathrm{~cm}$ and a full crossing angle of $\Theta_{c}=590 \mu \mathrm{rad}$ $\left(\Theta_{c}^{\text {norm }}=12.5 \sigma\right)$, the above correction method has been applied in three different cases, namely: compensating for (i) the $c_{p q}^{\mathrm{LR}}$ coefficients $(4,0)$ and $(6,0)$ [and therefore also $(0,4)$ and $(0,6)$ by symmetry], (ii) $(4,0)$ and $(8,2)$ [and therefore also $(0,4)$ and $(2,8)]$, and finally (iii) $(2,2)$ and $(5,5)$. In the three cases, a common goal is to cancel out some fourth order RDTs, in fact the direct anharmonicity coefficients $\partial Q_{x} / \partial J_{x} \equiv \partial Q_{y} / \partial J_{y}$ in cases (i) and (ii) and the cross-anharmonicity term $\partial Q_{x} / \partial J_{y} \equiv \partial Q_{y} / \partial J_{x}$ in case (iii). These quantities are indeed the first nonzero detuning terms driven by the LR beam-beam interactions, with no intrinsic self-compensation for the (HL-)LHC baseline configuration (see Sec. II B). While for the first two cases, the priority is clearly to shape the tune footprint on the antidiagonal (see later the "wings" of typical tune footprints before correction, which concern particles oscillating in the horizontal or vertical planes, with $J_{y} \sim 0$ or $J_{x} \sim 0$, respectively), case (iii) is concentrated onto the correction of the nonlinear dynamics at 45 degrees in the betatron phase space, i.e., for particles with $J_{x} \equiv J_{y}$. Then while the focus is put on RDTs of higher order (tenth order terms) in the last two cases, the sixth order terms have been chosen in case (i), although in the three cases the corresponding detuning terms are self-compensated in the alternated crossing scheme (and round optics). Finally it is worth noting that the $(5,5)$ RDT selected in case (iii) is not physically excited by the LR beam-beam interactions in the case of purely horizontal or vertical crossing. Nevertheless, as for any other "physical" RDTs, it constitutes a welldefined quantity, or "mathematical" norm, which can a priori be used for the correction. In all cases, one will show later that for specific aspect ratio of the $\beta$-functions at the wire location, the wire settings (current and transverse positions) do no longer depend on the correction type, more precisely on the selection of the two or four "mathematical" RDTs which are considered in the correction algorithm.

Wire current.-For a maximum beam intensity of $N_{0}=$ $2.2 \times 10^{11}$ protons per bunch [2], each LR beam-beam interaction corresponds to an equivalent integrated current of $(I L)_{\text {eq }}=10.56 \mathrm{~A} \mathrm{~m}$ [see Eq. (9)]. This would lead to an integrated current of about $190 \mathrm{~A}$ m for each of the two wires if the assumed $N_{\mathrm{LR}}=18 \mathrm{LR}$ interactions per IP side could be combined in a strictly additive way, for instance if these interactions would take place at the same normalized separation and at constant $\beta$ aspect ratio, which is not the case as previously discussed. In order to study in detail the real situation, the integrated current needed in the two wires $\left[N_{w . \mathrm{L}}=N_{w . \mathrm{R}}=N_{w}\right.$ in Eq. (18)] is plotted in Fig. 5 as a function of the position of the wire with respect to the IP,

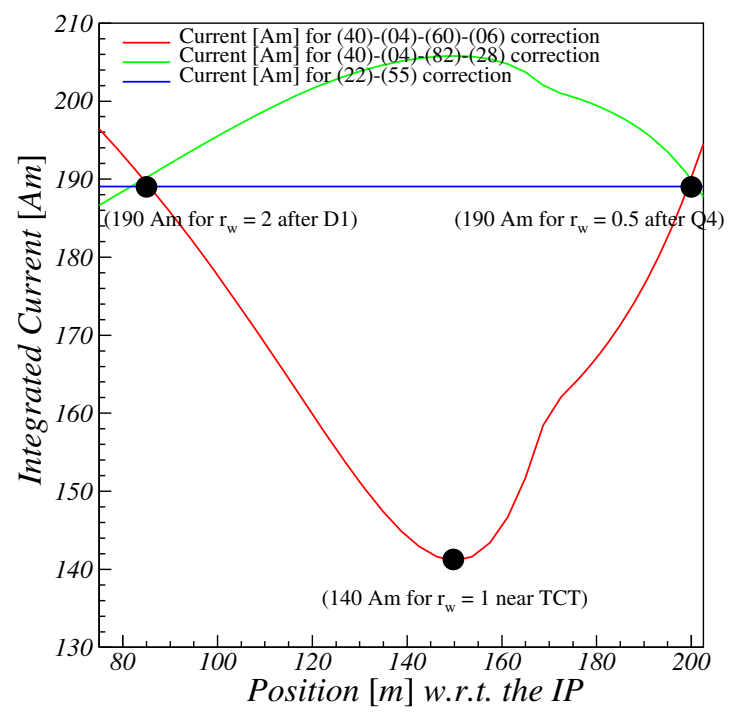

FIG. 5. Integrated current needed in the left and right wires as a function of the wire position with respect to the IP, assuming $N_{\mathrm{LR}}=18 \mathrm{LR}$ beam-beam interactions on either side of the IP and a beam intensity of $2.2 \times 10^{11}$ protons per bunch. Three different types of correction have been considered as described in the text.

and therefore implicitly as a function of the $\beta$ function aspect ratio $r_{w}$ at the wire location. This current is constant, and close to the expected value of $190 \mathrm{~A}$ m only in the third case (iii), as expected from Eq. (18) when $p_{1}=q_{1}$ and $p_{2}=q_{2}$. The situation is very different in the other two cases where the current needed in the wires can substantially vary with the aspect ratio $r_{w}$. For an aspect ratio of $r_{w} \sim 1$ for instance, the current needed is further pushed in case (ii) while it is substantially decreased in case (i). In any case we will see later that an aspect ratio of one at the wire location always correspond to the worst case in terms of the quality of the correction, i.e., for the driving terms which have not been selected in the correction process. A remarkable feature is however that for an aspect ratio close to two or one half, the current needed is almost the same in the three cases.

Transverse position $d_{w}$. -Figure 6 shows the transverse distance of the left and right wires with respect to the weak beam $\left[d_{w . \mathrm{L}}=d_{w . \mathrm{R}}=d_{w}\right.$ in Eq. (18)] as a function of the wire position with respect to the IP. These distances are given in $\mathrm{mm}$ in Fig. 6(a), in which case they are the same for the left and right wires and do not depend on the orientation of the crossing plane. When normalized by the size of the weak beam in the crossing plane, as in Fig. 6(b), these quantities are no longer the same for the left and right wires (except for an aspect ratio $r_{w}=1$ ), but still fulfill a certain symmetry of exchange when passing from an horizontal to a vertical crossing angle. Finally, it is worth noting that the same feature as above is reproduced, where an aspect ratio of $r_{w} \sim$ $1 / 2$ or $r_{w} \sim 2$ at the wire location leads to the same setting for the three correction types under consideration. 


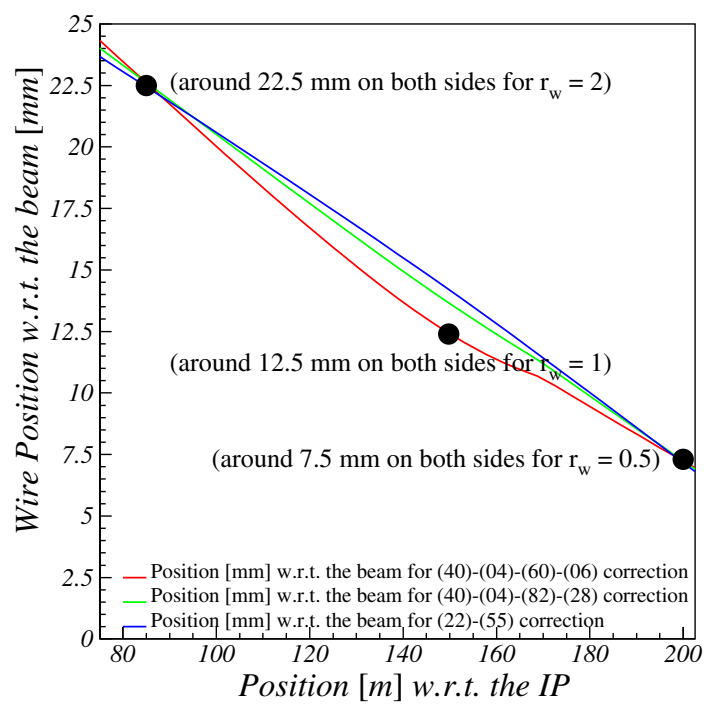

(a)

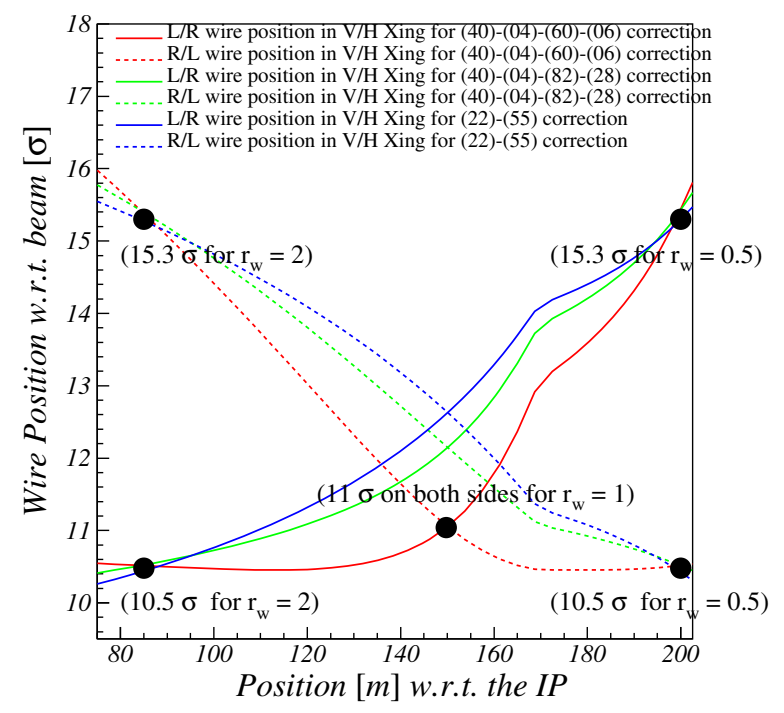

(b)

FIG. 6. Transverse distance of the left and right wires with respect to the weak beam assuming an horizontal or vertical crossing angle of $590 \mu \mathrm{rad}$. These distances are given as a function of the wire location with respect to the IP, reported in mm in (a) and normalized in (b) by the size of the weak beam in the crossing plane (using the intermediate optics and layout version SLHCV3.1b of the HL-LHC [23] with $\beta_{x, y}^{*}=15 \mathrm{~cm}$, and assuming a normalized emittance of $\gamma \epsilon=2.5 \mu \mathrm{m}$ ).

It becomes then legitimate to conjecture the existence of an optimal aspect ratio(s) for the $\beta$ functions at the wire location, for which the correction of only two (or four) resonance driving terms would automatically minimize all the others. This remarkable property becomes obvious when looking at the residual coefficients $c_{p q}^{\mathrm{LR}}+c_{p q}^{w . \mathrm{L}}+$ $c_{p q}^{w . R}$ after correction, as reported in Fig. 7, directly as a function of the aspect ratio $r_{w}$ and for the three correction types envisaged above. While for each correction type and regardless of the aspect ratio $r_{w}$ the wires can in principle be adjusted in current and transverse position in order to exactly compensate two (or four) coefficients, the other coefficients are in general very poorly controlled, with very large excursion and a worst case, which is systematically reached around $r_{w} \sim 1$. On the other hand all these terms can be strongly and simultaneously diminished, regardless of the correction type, when the aspect ratio approaches the two optimal values of $r_{w} \sim 2$ (right after D1) and $r_{w} \sim 1 / 2$ (in the zone D2/Q4 or even beyond depending on the triplet layout and optics matching). First of all the fact that the product of these two optimal aspect ratios is equal to unity is not a hazard. This property results from the optics antisymmetry of the (HL-)LHC. More precisely the sum occurring in Eq. (14) is invariant by exchanging $x$ into $y$, which correspond to an exchange of $r_{w}$ into $1 / r_{w}$ at the wire on the right, and conversely for the wire on the left of the IP, for the same result in terms of RDT residuals after correction. On the other hand the nontrivial result is that this sum can be accurately approximated by only two nonlinear kicks, and this for a large selection of $(p, q)$ coefficients, at least up to the order $p+q=10$ as showed in Fig. 7. The authors have presently no analytical proof of this numerical evidence. In order to invalidate the possibility that this property could be specific to the layout of the HL-LHC triplet, the correction method has also been tested on the existing LHC triplet which is shorter, with only $N_{\mathrm{LR}}=15 \mathrm{LR}$ beam-beam interactions per IP side, 6 of them taking place in the drift space between the IP and the first quadrupole Q1, i.e., at constant normalized separation and $\beta$ aspect ratio. Also in this case the above property has been reproduced with the only difference that the optimal aspect ratios have been found to be slightly shifted, equal to $r_{w} \sim 0.6$ and $r_{w} \sim 1.7$.

Another important conclusion from Fig. 7 is that, for a nonoptimal positioning of the wires, a correction algorithm involving high order RDTs (as in cases (ii) and (iii)) seems to give better results for the residuals of the nonselected RDTs after correction [e.g., compare Figs. 7(ii) and 7(i) at an aspect ratio $\left.r_{w} \sim 1\right]$. This conclusion has also been confirmed by dedicated dynamic aperture studies performed on the HL-LHC alternative scenario with flat optics (see Sec. III C) and wires installed at $r_{w} \sim 1$. Nevertheless, since the position corresponding to an aspect ratio of $r_{w} \sim 0.5$ is much more suitable for integration in the (HL-)LHC, the worst case corresponding to $r_{w} \sim 1$ will no longer be considered in the rest of the paper.

Before closing this chapter, it remains important to analyze a posteriori the behavior of the exact driving terms after correction by taking into account the phase shifts, although small, between the wire location and the LR beam-beam encounters, namely: 

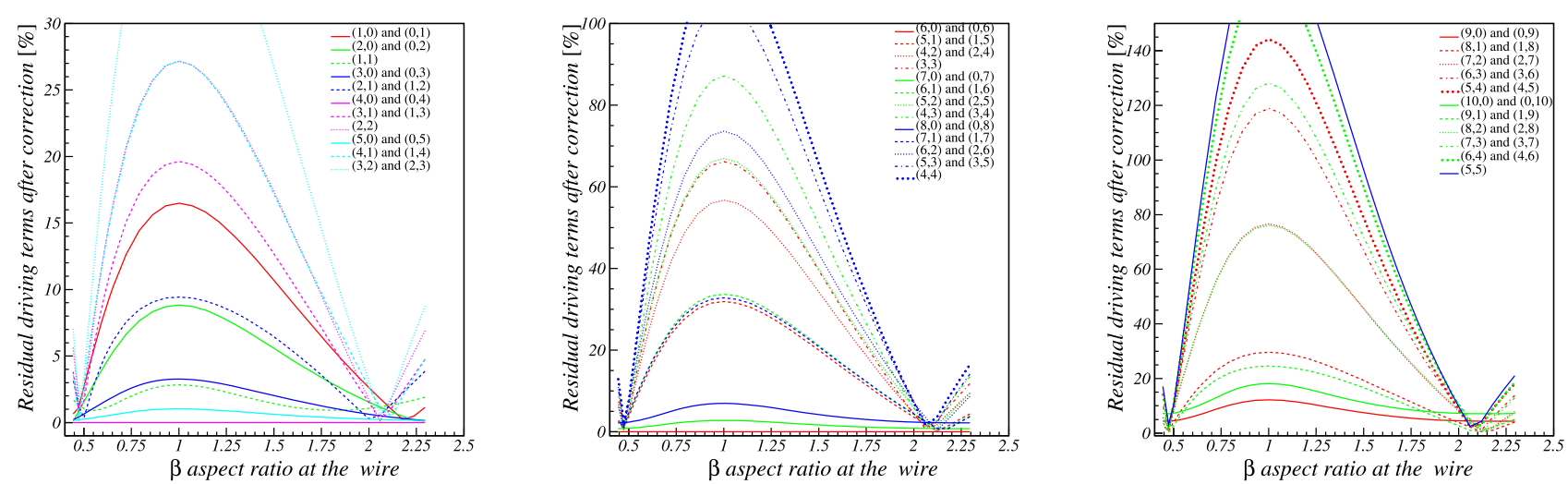

(i) Residual coefficients $c_{p q}^{L R}$ after correction, up to the 10th order, for the correction type (40)-(04)-(60)-(06)
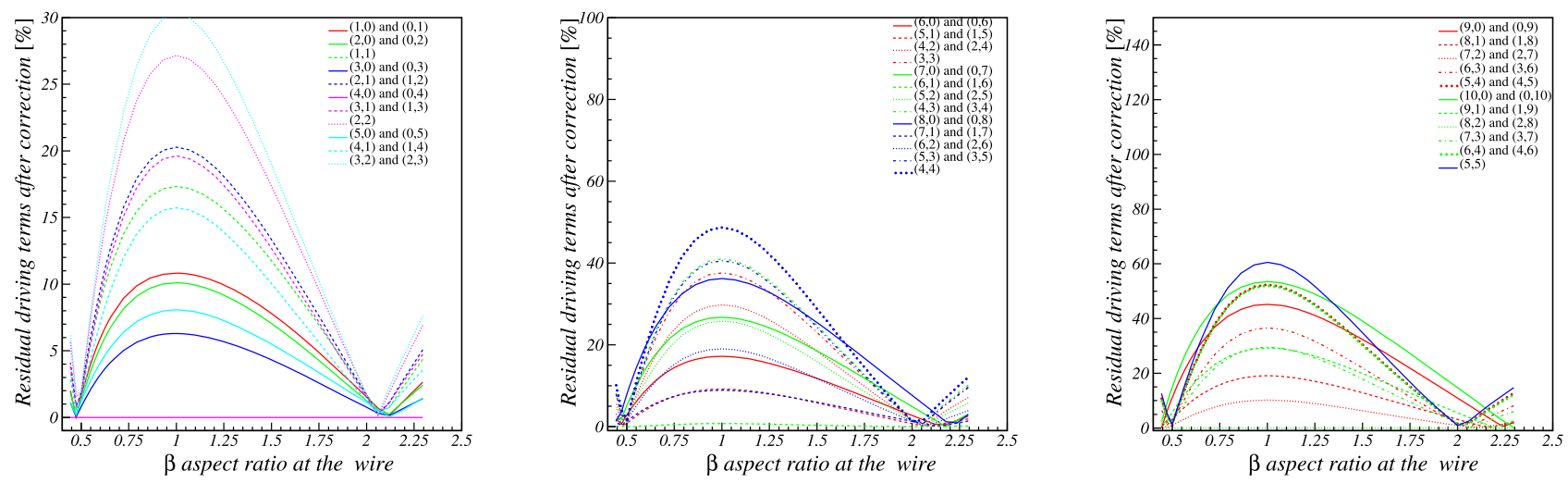

(ii) Residual coefficients $c_{p q}^{L R}$ after correction, up to the 10th order, for the correction type (40)-(04)-(82)-(28)
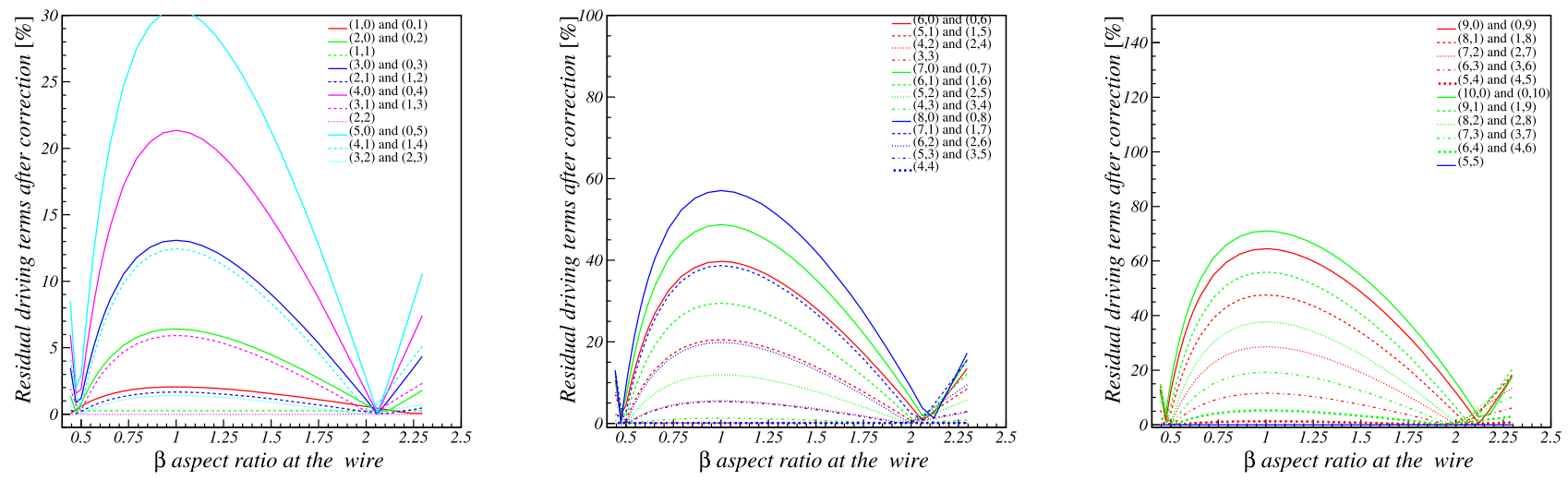

(iii) Residual coefficients $c_{p q}^{L R}$ after correction, up to the 10th order, for the correction type type (22)-(55)

FIG. 7. Residual coefficients $c_{p q}^{\mathrm{LR}}+c_{p q}^{w . \mathrm{L}}+c_{p q}^{w . \mathrm{R}}$ after correction reported in percent of their value before correction [see Eqs. (14) and (15)], given up to 10th order $(p+q \leq 10, p \geq 0, q \geq 0)$, and shown as a function of the aspect ratio $r_{w}$ at the wire position. Three possible correction algorithms are envisaged as described in the text.

$$
\tilde{c}_{p q}^{\mathrm{LR}} \equiv \sum_{k \in \mathrm{LR}} \frac{\beta_{x}^{|p| / 2}\left(s_{k}\right) \beta_{y}^{|q| / 2}\left(s_{k}\right)}{d_{b b}^{|p|+|q|}\left(s_{k}\right)} e^{i\left[p \mu_{x}\left(s_{k}\right)+q \mu_{y}\left(s_{k}\right)\right]},
$$

with similar definitions for the RDTs $\tilde{c}_{p q}^{w . \mathrm{L} / \mathrm{R}}$ induced by the left and right wires. As described in Sec. II B, for horizontal (resp. vertical) crossing, only the RDTs $\tilde{c}_{p q}^{\mathrm{LR}}$ with $q$ even (respectively $p$ even) are excited. In this case it is also relevant to distinguish between the coefficients $(p, q)$ and $(p,-q)$ with $p \geq 0$ and $q \geq 0$. Finally for the LHC optics antisymmetry, it is easy to see that when a coefficient $(p, q)$ is excited in horizontal crossing, the modulus of the $(q, p)$ coefficient is the same in vertical crossing. This being said, even for $\beta^{*}$ as small as $15 \mathrm{~cm}$, the betatron phase shift can 

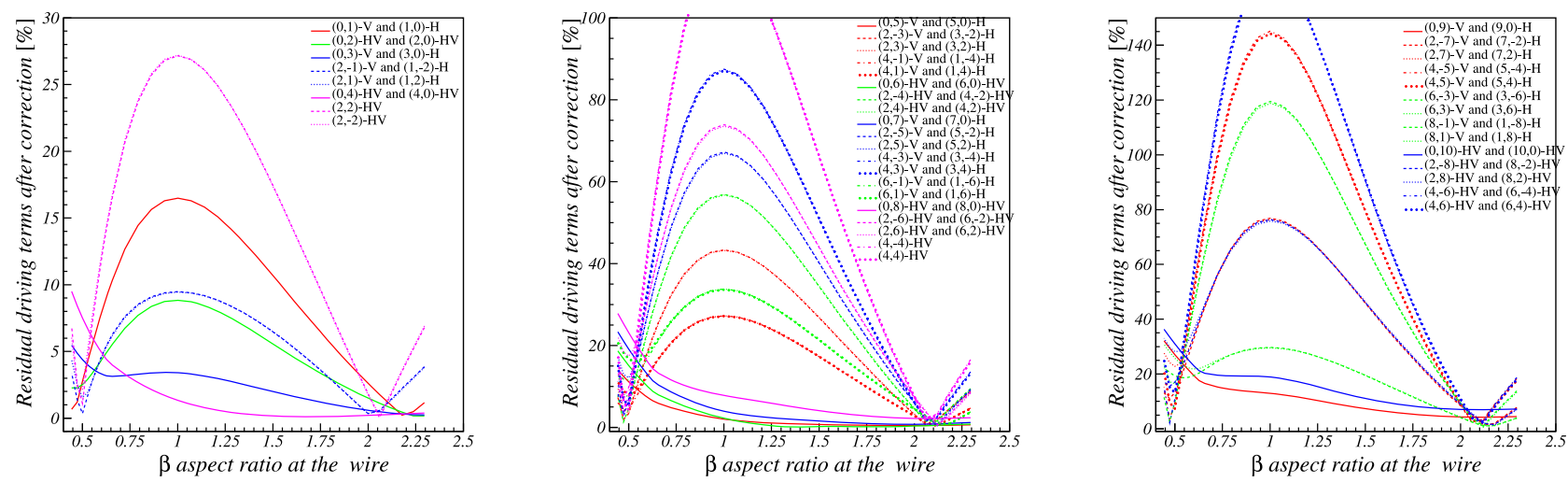

(i) Residual driving terms $\tilde{c}_{p q}^{L R}$ after correction, up to the 10th order, for the correction type (40)-(04)-(60)-(06)
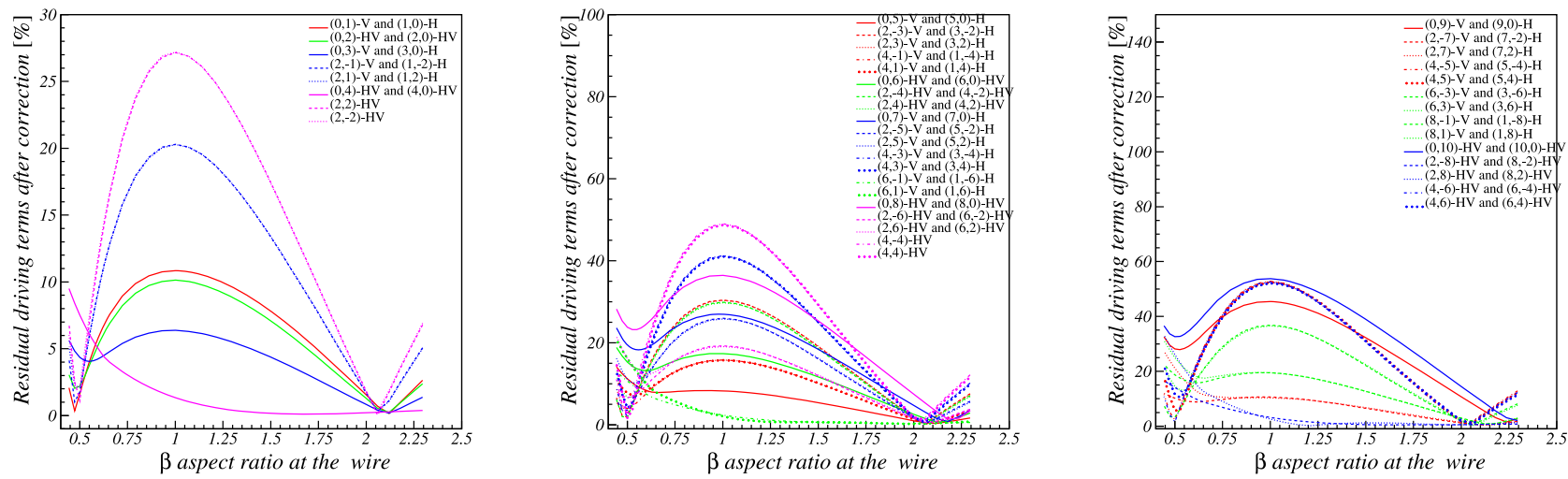

(ii) Residual driving terms $\tilde{c}_{p q}^{L R}$ after correction, up to the 10th order, for the correction type (40)-(04)-(82)-(28)
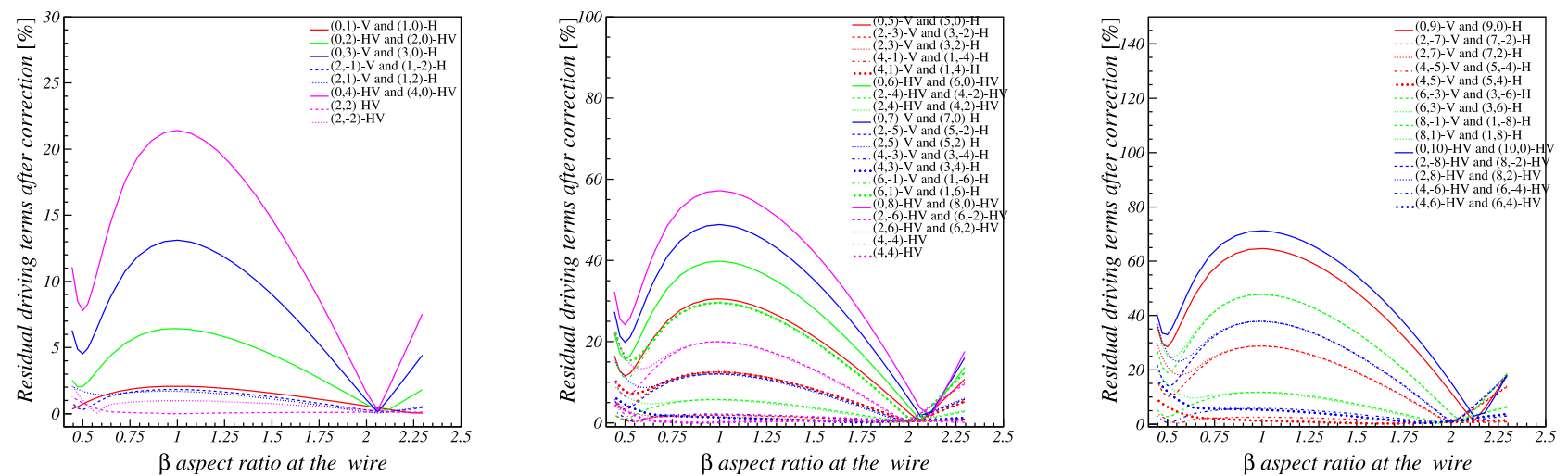

(iii) Residual driving terms $\tilde{c}_{p q}^{L R}$ after correction, up to the 10th order, for the correction type (22)-(55)

FIG. 8. Residual driving terms $\tilde{c}_{p q}^{\mathrm{LR}}+\tilde{c}_{p q}^{w . \mathrm{L}}+\tilde{c}_{p q}^{w . \mathrm{R}}$ after correction reported in percent of their value before correction [see Eq. (21)], given up to the 10th order $(p+|q| \leq 10, p \geq 0)$, and shown as a function of the aspect ratio $r_{w}$ of the $\beta$ functions at the wire position. Three possible correction algorithms are envisaged as described in the text. The notation (p,q)-V [respectively (q,p)-H] stands for the $(p, q)$ driving term excited in the presence of a vertical (respectively horizontal) crossing angle of $590 \mu \mathrm{rad}$. Despite $\beta^{*}$ is $15 \mathrm{~cm}$ in both planes, the small but nonzero phase shifts between the wires and the LR encounters clearly impact on the quality of the correction at the second optimal aspect ratio of $r_{w} \sim 0.5$.

possibly reach up to 4 degrees between the LR beam-beam interactions and a position at $\pm 200 \mathrm{~m}$ from the IP [see Fig. 4(b)]. As a result and as shown in Fig. 8, while the property of simultaneous minimization of all RDTs is well preserved at the first optimal aspect ratio of $r_{w} \sim 2$ taking place right after D1, some net degradations can be observed, in particular for the high order driving terms, at the second optimal aspect ratio of $r_{w} \sim 0.5$ (situated at 
$\sim \pm 200 \mathrm{~m}$ from the IP). Nevertheless, this second optimal position still remains interesting for the detuning terms, even for the exact RDTs with respect to the situation which would be obtained at $r_{w} \sim 1$, but also for integration in the case of the (HL-)LHC.

\section{ILLUSTRATIONS}

This section describes several possible HL-LHC variants, each of them presenting a series of advantages, but all assuming the implementation of current bearing wires (or e-lenses) for the compensation of the long-range beambeam interactions. The pros and cons of each variant will only be briefly mentioned. We will rather analyze in great detail the beneficial effects of the LR beam-beam compensation, in terms of the tune footprint and/or frequency map analysis (FMA) $[26,27]$ calculated with the code LIFETRAC [28], and/or long term (1,000,000 turns) dynamical aperture (DA), i.e., the largest region of the transverse physical space where a particle is stable after a given number of turns, as calculated with the code SIXTRACK [29].

Table I summarizes the main parameters and key quantities to assess the HL-LHC performance in each of these configurations, together with the wire settings which have been used in each case.

We will start with the HL-LHC baseline as the reference case, in particular with a normalized crossing angle of
$\Theta_{c}^{\text {norm }}=12.5 \sigma(590 \mu \mathrm{rad})$ and an horizontal and vertical (HV) alternated crossing angle in the two high luminosity insertions of the ring, IR1 and IR5, respectively. In the second case the only change is a reduction of the crossing angle down to $\Theta_{c}^{\text {norm }}=9.5 \sigma(450 \mu \mathrm{rad})$. In this configuration the performance of the machine is strictly preserved but a $25 \%$ reduction of the nominal rf deflecting field in the crab-cavities becomes possible. The line density of events per bunch crossing (pile up) is also slightly improved, the radiation dose deposited in the inner triplet is generally mitigated at lower crossing angle, and the strength requested for the IR correctors is obviously reduced for crossing bumps of smaller magnitude. These form a series of beneficial effects to be kept in mind when reducing the crossing angle.

The third and fourth cases refer to possible variants of the HL-LHC baseline with an HH or VV crossing scheme in IR1 and IR5 (and the crab-cavity deflecting field reoriented accordingly). Here the concern is related to collimation aspects of the machine section with respect to the outgoing beam and/or to the lifetime of the inner triplet quadrupoles. First of all, a vertical crossing angle in both low- $\beta$ insertions (VV scheme) would have the advantage to simplify the design of several beam absorbers in the matching section, which aims at protecting its magnets from the debris coming from the IP, and neutral debris in

TABLE I. Baseline parameters of the HL-LHC (25 ns bunch spacing version [30]) and comparison with other possible variants and an alternative with flat optics and no crab-cavities. The corresponding wire settings are indicated in each case (calculated for the SLHCV3.1b optics and layout version of the HL-LHC). The Performance related quantities, such as virtual luminosity, leveling time, or line pile-up density have been calculated following Ref. [31].

\begin{tabular}{|c|c|c|c|c|c|}
\hline Parameters and Layout & Baseline & $\begin{array}{c}\text { Baseline with } \\
\text { smaller } \Theta_{c}\end{array}$ & $\begin{array}{l}\text { Baseline with } \\
\text { HH scheme }\end{array}$ & $\begin{array}{l}\text { Baseline with } \\
\text { VV scheme }\end{array}$ & $\begin{array}{c}\text { Alternative with } \\
\text { flat optics }\end{array}$ \\
\hline Crab-cavity & Yes & Yes & Yes & Yes & No \\
\hline Crab-cavity voltage $[\mathrm{MV}]$ / beam /IP side & 12 & 9 & 12 & 12 & - \\
\hline Crossing orientation in IR $1 / 5$ & $\mathrm{~V} / \mathrm{H}$ & $\mathrm{V} / \mathrm{H}$ & $\mathrm{H} / \mathrm{H}$ & $\mathrm{V} / \mathrm{V}$ & $\mathrm{V} / \mathrm{H}$ \\
\hline Optics type & Round & Round & Round & Round & Flat \\
\hline Energy $[\mathrm{TeV}]$ & 7 & 7 & 7 & 7 & 7 \\
\hline Bunch spacing $[\mathrm{ns}]$ & 25 & 25 & 25 & 25 & 25 \\
\hline Number of bunches colliding at IP1 and IP5 & 2736 & 2736 & 2736 & 2736 & 2736 \\
\hline Bunch charge $\left[10^{11}\right]$ & 2.2 & 2.2 & 2.2 & 2.2 & 2.2 \\
\hline Bunch length $[\mathrm{cm}]$ & 7.50 & 7.50 & 7.50 & 7.50 & 10.0 \\
\hline Normalized emittance $[\mu \mathrm{m}]$ & 2.5 & 2.5 & 2.5 & 2.5 & 2.5 \\
\hline$\beta_{\mathrm{X}}^{*} / \beta_{\|}^{*}[\mathrm{~cm}]$ & $15 / 15$ & $15 / 15$ & $15 / 15$ & $15 / 15$ & $40 / 10$ \\
\hline Full crossing angle $[\mu \mathrm{rad}]$ & 590 & 450 & 590 & 590 & 300 \\
\hline Beam separation $[\sigma]$ & 12.5 & 9.5 & 12.5 & 12.5 & 10.4 \\
\hline Wire current [A] for 18 LR's (Left and Right) & 192 & 192 & 192 & 192 & 192 \\
\hline Wire position [mm] w.r.t. the beam (L and R) & 7.6 & 5.8 & 7.6 & 7.6 & 3.9 \\
\hline$\beta[\mathrm{m}]$ at the wire in the crossing-plane $(\mathrm{L} / \mathrm{R}$ or $\mathrm{R} / \mathrm{L})$ & $1540 / 750$ & $1540 / 750$ & $1540 / 750$ & $1540 / 750$ & $575 / 280$ \\
\hline Normalized wire position $[\sigma](\mathrm{L} / \mathrm{R}$ or $\mathrm{R} / \mathrm{L})$ & $10.6 / 15.2$ & $8.1 / 11.6$ & $10.6 / 15.2$ & $10.6 / 15.2$ & $8.8 / 12.6$ \\
\hline Max. head-on beam-beam tune shift (3 IPs) & 0.032 & 0.032 & 0.032 & 0.032 & 0.025 \\
\hline Virtual luminosity $\left[10^{35} \mathrm{~cm}^{-2} \mathrm{~s}^{-1}\right]$ & 19.6 & 20.2 & 19.6 & 19.6 & 10.1 \\
\hline Pile up events per bunch crossing at $5 \times 10^{34} \mathrm{~cm}^{-2} \mathrm{~s}^{-1}$ & 138 & 138 & 138 & 138 & 138 \\
\hline Peak line pile up density $\left[\mathrm{mm}^{-1}\right]$ at $5 \times 10^{34} \mathrm{~cm}^{-2} \mathrm{~s}^{-1}$ & 1.25 & 1.21 & 1.25 & 1.25 & 1.38 \\
\hline Leveling time $[\mathrm{h}]$ at $5 \times 10^{34} \mathrm{~cm}^{-2} \mathrm{~s}^{-1}$ & 8.3 & 8.4 & 8.3 & 8.3 & 4.9 \\
\hline Integrated luminosity $\left[\mathrm{fb}^{-1}\right]$ after $10 \mathrm{~h}$ & 1.75 & 1.75 & 1.75 & 1.75 & 1.54 \\
\hline
\end{tabular}


particular. In the case of horizontal crossing, the trajectories of the neutral debris are indeed relatively close to that of the circulating beam at the level of the D2 recombination dipole, which makes their collimation very challenging. On another perspective, an horizontal crossing scheme in both low- $\beta$ insertions (HH scheme) would reduce the integrated radiation dose taken by the inner triplet, and therefore would increase its lifetime due to the fact that the first inner triplet quadrupole Q1 is always focusing for the outgoing beam in the case of both IR1 and IR5 in the LHC. On the other hand, combining the VV scheme with a regular change of the crossing angle polarity (which is not possible in $\mathrm{H}$ crossing due to constraints imposed by the ring geometry) would also substantially improve the lifetime of the inner triplet [32], which certainly makes the VV configuration very attractive for the (HL-)LHC.

Finally, an alternative HL-LHC will be analyzed, as a backup plan in case of unavailability of the crab-cavities. This fifth and last case relies on flat optics with a strongly reduced crossing angle in the plane of larger $\beta^{*}\left(\Theta_{c}^{\text {norm }}=300 \mu \mathrm{rad}\right)$ in order to preserve the machine performance.

For each of these cases, the correction algorithm will target the compensation of the $(4,0),(0,4),(8,2)$ and $(2,8)$ driving terms. The wires will be assumed to be installed at a $\beta$ function aspect ratio of $r_{w}=0.5$ in between Q4 and Q5 (see Fig. 2), which makes the integration certainly easier, with the two beams already occupying two different beam pipes at this location, and generally less severe space constraints in this area. All the simulations have been based on the intermediate version SLHCV3.1b of the HL-LHC optics and layout [23], with in particular a maximum possible number of 18 LR beam-beam interactions (for a bunch spacing by $25 \mathrm{~ns}$ ) on either side of the IP1 and IP5 until the D1 separation dipole. All simulations have been performed at maximum bunch intensity $\left(N_{b}=2.2 \times 10^{11}\right.$ protons/ bunch) and minimum collision $\beta^{*}$, with head-on collision at zero or moderate Piwinsky angle in three experimental insertions, i.e., taking into account the LHCb insertion in IR8. Indeed, the LHCb experiment is supposed to run in the HL-LHC era [33], although at moderate luminosity (large $\beta^{*}$ of a few meters) but still contributing to the head-on beambeam tune shift as any of the two high luminosity insertions. Finally, the linear chromaticity was matched to 2 units with the arc sextupoles, the Landau octupoles were switched off in the arcs, and the field imperfections of the superconducting (HL-)LHC magnets were not taken into account (although a reduction of the crossing angle would also be beneficial in this respect).

\section{A. HL-LHC baseline with nominal or reduced crossing angle}

\section{Nominal bunches with maximal number of LR beam-beam interactions}

The baseline crossing angle has been chosen to $590 \mu \mathrm{rad}$ in the new high luminosity insertions of the HL-LHC, in order to warrant a dynamic aperture (DA) of $6 \sigma$, even in the extreme case of full beam current $\left(2.2 \times 10^{11} /\right.$ bunch $)$ and minimum $\beta^{*}(15 \mathrm{~cm})$, corresponding to the full virtual luminosity of $\sim 2 \times 10^{35} \mathrm{~cm}^{-2} \mathrm{~s}^{-1}$. With the wires switched on, the DA rises up to $8 \sigma$ for the nominal bunches undergoing the maximal number of LR interactions in IR1 and IR5 (see later in Sec. III A 2 the discussion on Pacman bunches). The wires therefore clearly open up the possibility to further reduce the crossing angle, even down to $450 \mu \mathrm{rad}$ [see Fig. 9(b)], owing to the mitigation of some LR beam-beam driven resonances in the horizontal plane (see Figs. 10 and 11).

\section{Pacman bunches}

The nominal (HL-)LHC filling scheme is made of several trains of 72 bunches spaced by $25 \mathrm{~ns}$, each train being spaced by a certain multiplier of $25 \mathrm{~ns}$ (gaps) which is determined by the rise times of the various injection and extraction kickers and by the circumference of the intermediate machines (PS, SPS) along the LHC injector chain (see e.g., [30]). In addition, an abort gap with a duration of about $3 \mu$ s is left free of bunches in the filling pattern of the LHC beam in order to accommodate for the rise time of the LHC extraction kicker. As a result not all the bunches experience the same number of LR beam-beam interactions, in particular in the high luminosity insertions IR1 and IR5, leading to the so-called "Pacman effect" with different bunches showing slightly different LR beambeam induced closed orbit, tune shift, or tune spread. While the number of LR beam-beam interactions is maximal for the nominal bunches located well inside the trains, it is reduced for the bunches at the head or in the tail of the trains, with a number of interactions which is reduced on the left and on the right, respectively, of the interaction points. This effect is obviously the most pronounced for the first bunch of the first train right after the abort gap. For Beam1, this bunch indeed misses all the LR interactions on the left side of IP1 and IP5, and conversely for the last bunch of the last train before the abort gap (and conversely for Beam2). These two extreme bunches will be called the PacmanL and PacmanR bunches in the following. Due to the HV alternated crossing configuration in IR1 and IR5, and the symmetric positioning of these two insertions in the LHC ring, the PacmanL and PacmanR bunches and, in general, all the Pacman bunches which undergo a nonmaximal number of LR beam-beam interactions are however not shifted in betatron tune with respect to the nominal bunches. Therefore, not only the contribution of the LR interactions to the tune spread should be smaller for the Pacman bunches, but also the dynamic aperture should be larger for the particles belonging to these specific bunches. In order to preserve this situation, an overcompensation of the LR beam-beam interactions seen by the Pacman bunches should a priori be avoided. This means that the current in the wires (or e-lenses) should be modulated in 


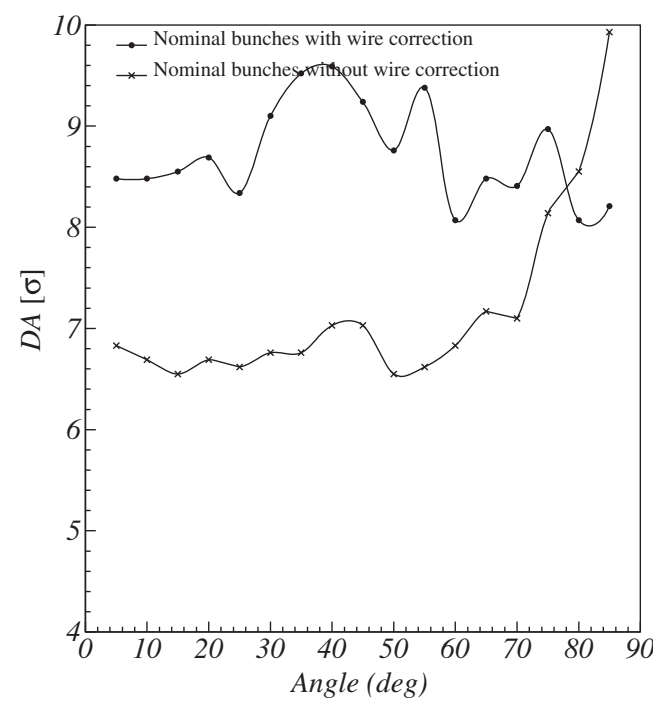

(a)

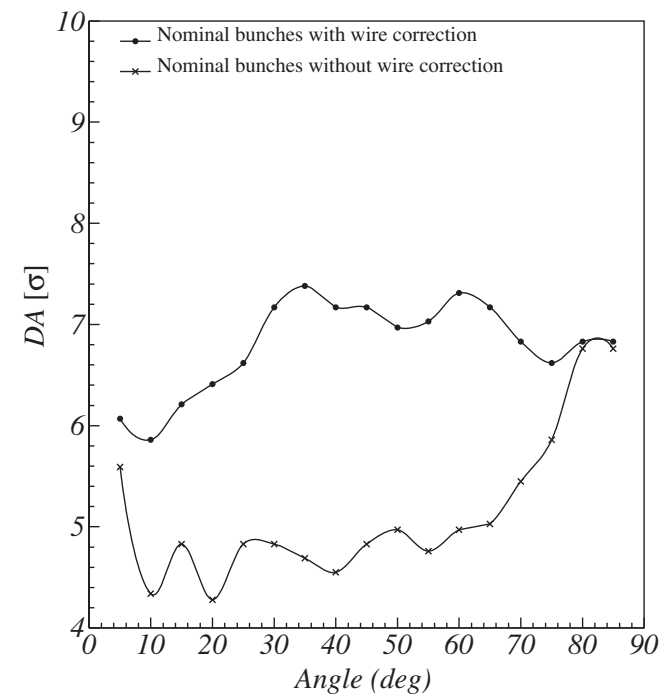

(b)

FIG. 9. Nominal bunches and HL-LHC baseline configuration at nominal [(a) with $\left.\Theta_{c}=590 \mu \mathrm{rad}\right]$ or reduced [(b) with $\Theta_{c}=450 \mu \mathrm{rad}$ ] crossing angle, corresponding to the first and second column of Table I: 1,000,000 turns dynamic aperture in collision, as a function of the phase space angle, with or without LR compensation, expressed in terms of beam sigma. Each particle is tracked with an initial momentum offset of $2.7 \times 10^{-4}$ corresponding to two thirds of the LHC rf bucket half-height at 7 TeV.

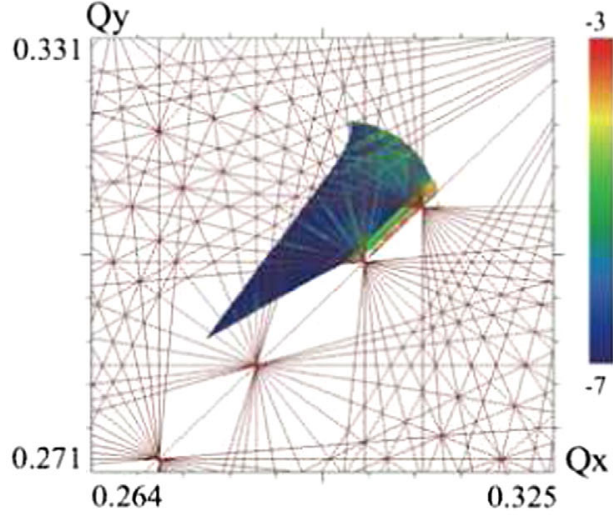

(a)

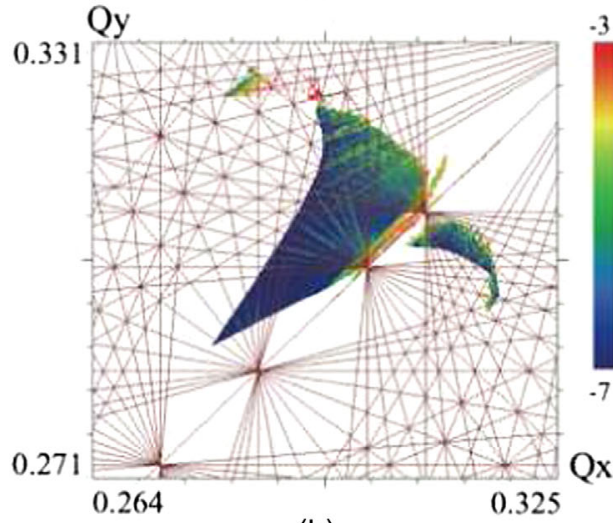

(b)

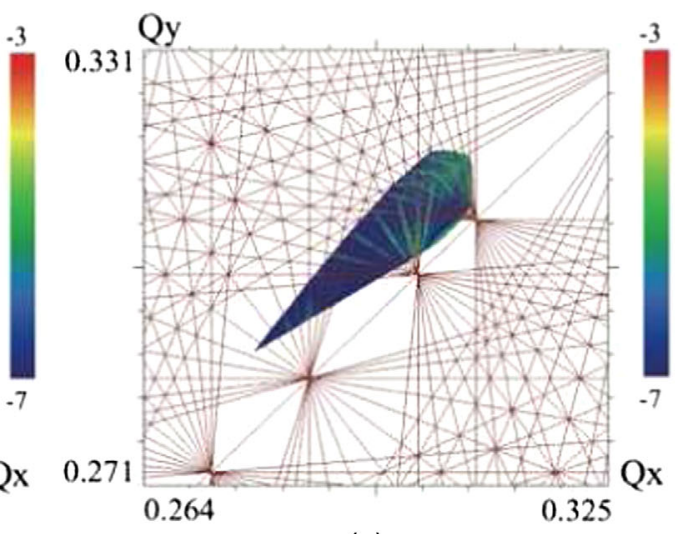

(c)

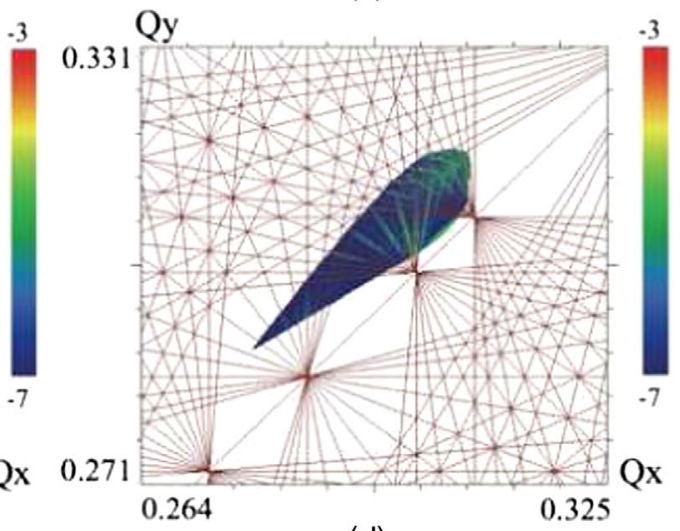

(d)

FIG. 10. Nominal bunches and HL-LHC baseline configuration at nominal [top (a) and (c) with $\Theta_{c}=590 \mu$ rad] or reduced [bottom (b) and (d) with $\Theta_{c}=450 \mu \mathrm{rad}$ ] crossing angle, corresponding to the first and second column of Table I: tune footprint for particles up to $6 \sigma$ betatron amplitude in collision with LR compensation [right (c) and (d)] or without [left (a) and (b)]. 


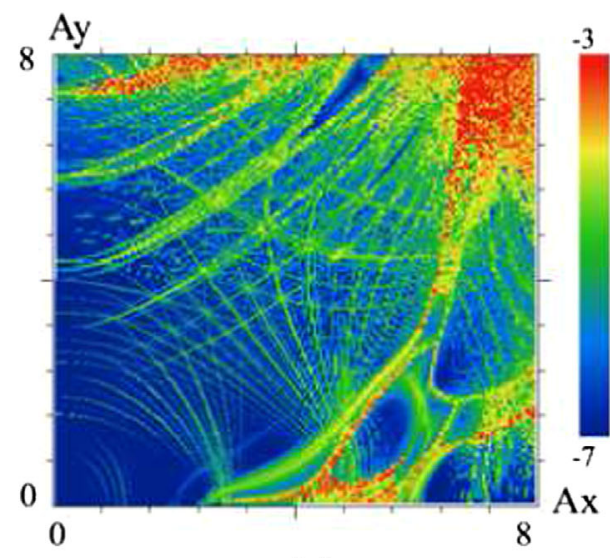

(a)

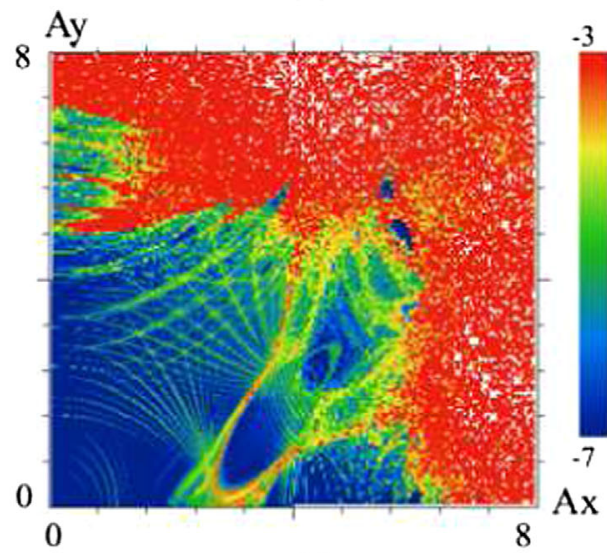

(b)

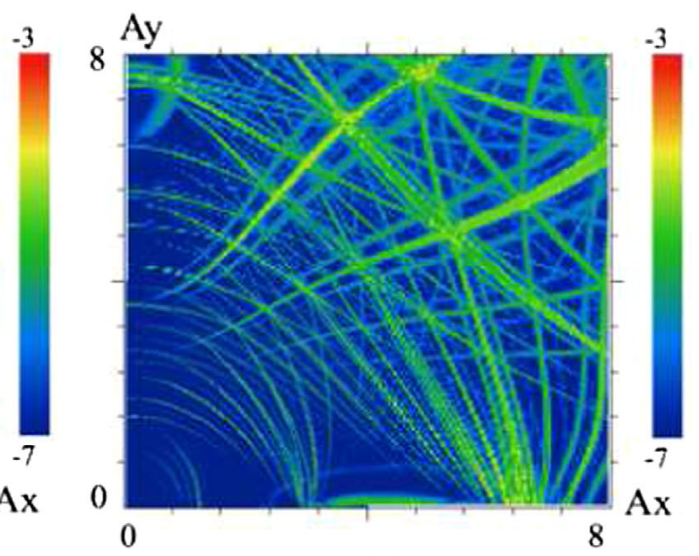

(c)

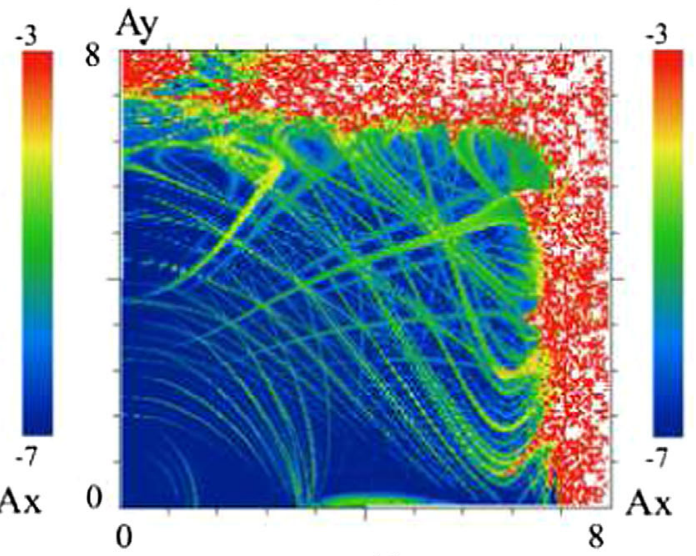

(d)

FIG. 11. Nominal bunches and HL-LHC baseline configuration at nominal [top (a) and (c) with $\Theta_{c}=590 \mu$ rad] or reduced [bottom (b) and (d) with $\Theta_{c}=450 \mu \mathrm{rad}$ ] crossing angle, corresponding to the first and second column of Table I: frequency diffusion maps for on-momentum particles up to $8 \sigma$ betatron amplitude in collision with [right (c) and (d)] or without [left (a) and (b)] LR compensation.

proportion with the number of LR interactions seen by each individual bunches (e.g., a reduction of current by a factor of 2 for the extreme PacmanL and PacmanR bunches), keeping constant the transverse position $d_{w}$ of the wires, as calculated for the nominal bunches with maximal and a left/ right symmetric number of LR beam-beam interactions. On the other hand, given the large difference in complexity between a dc and a pulsed system, this condition will need to be reassessed in detail depending on the machine configuration which will be decided and achievable in the end, but which also means that this option needs to stay open. The latter is certainly less needed for the baseline HV configuration at large crossing angle and nominal crabcavity voltage, but more and more at reduced crossing angle (and deficient crab-cavity voltage), and is certainly mandatory to avoid up to $\pm 5 \times 10^{-3}$ bunch to bunch tune shift in the case of the $\mathrm{HH}$ or VV crossing scheme that will be discussed later on. Assuming the reduced crossing angle of $450 \mu \mathrm{rad}$ and pulsed wires, the dynamic aperture of the PacmanL and PacmanR bunches is reported accordingly in Fig. 12, before and after compensation with the wires (at half of the current). As expected before correction, the DA is already better for these Pacman bunches than for the nominal bunches [compare Figs. 9(b) and 12(a)-(b)], but the $6 \sigma$ target is definitely met only after correction. A rapid inspection of the tune footprints for the PacmanL and PacmanR bunches confirms the benefit of the correction (see Fig. 13).

\section{B. HL-LHC variants with $\mathrm{HH}$ or $\mathrm{VV}$ crossing scheme}

As discussed in Sec. II B, the alternated HV crossing scheme of the (HL-)LHC has the advantage to establish the conditions for a self-compensation of the $(4 n+2)$-pole detuning terms induced by the LR beam-beam interactions in IR1 and IR5, and in particular the tune shift, assuming that the two low- $\beta$ insertions are running with the same round $\left(\beta_{x}^{*} \equiv \beta_{y}^{*}\right)$ collision optics. Furthermore, this selfcompensation is not only granted for the nominal bunches but also for the Pacman bunches, due to the fact that the topology of the LR interactions is exactly the same in IR1 and IR5 for a given arbitrary bunch. As a result, the other two possible crossing configurations, namely the $\mathrm{HH}$ and 


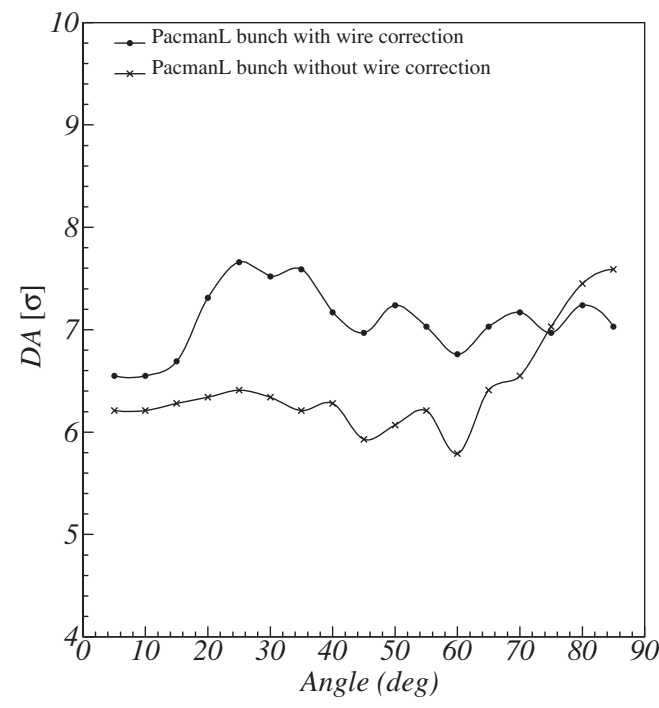

(a)

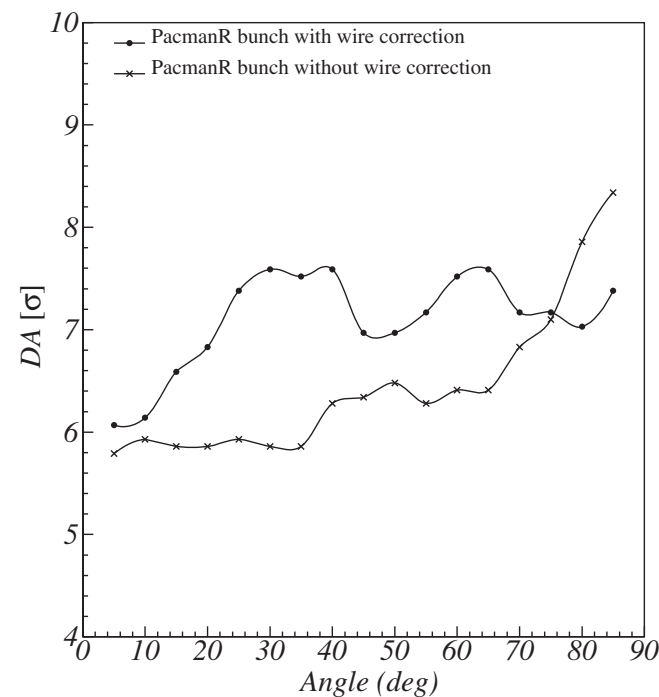

(b)

FIG. 12. PacmanL (a) and PacmanR (b) bunches, HL-LHC baseline configuration at reduced crossing angle $\left(\Theta_{c}=450 \mu \mathrm{rad}\right)$, corresponding to the second column of Table I: 1,000,000 turns dynamic aperture in collision, as a function of the phase space angle, with or without LR compensation, expressed in terms of beam sigma. Each particle is tracked with an initial momentum offset of $2.7 \times 10^{-4}$ corresponding to two thirds of the LHC rf bucket half-height at $7 \mathrm{TeV}$.

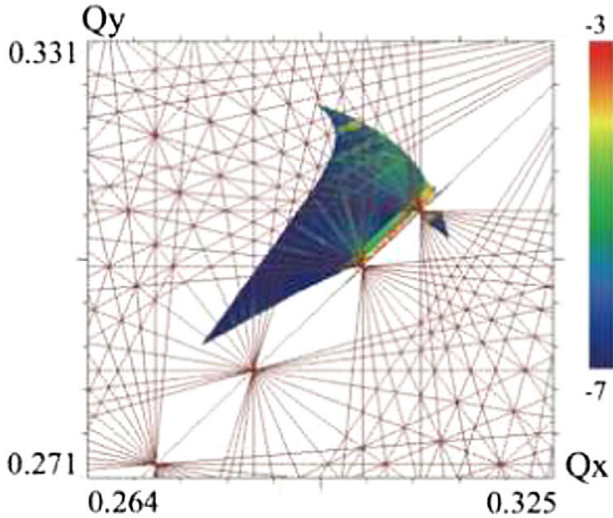

(a)

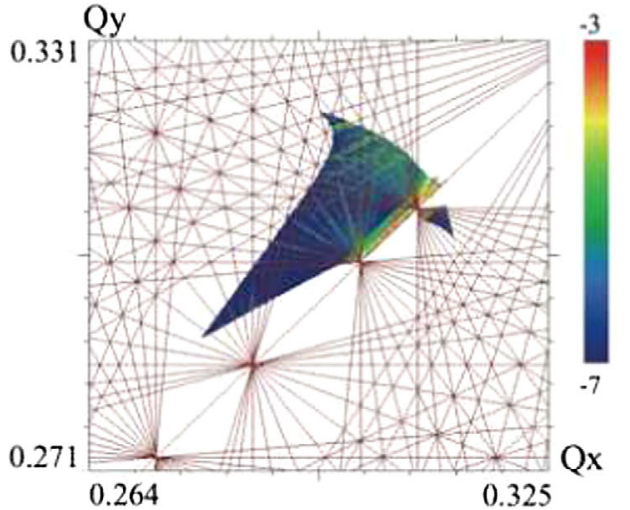

(b)

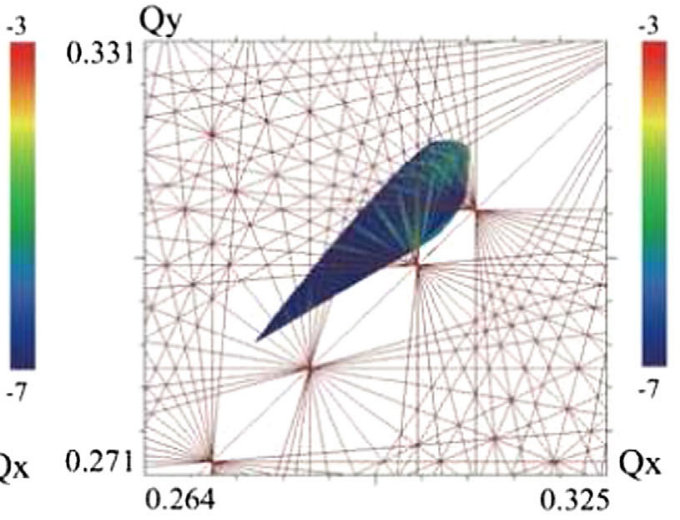

(c)
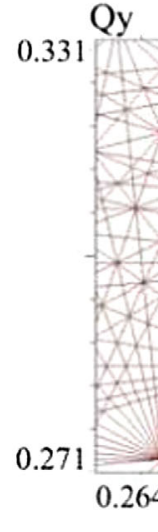

FIG. 13. PacmanL [top (a) and (c)] and PacmanR [bottom (b) and (d)] bunches, HL-LHC baseline configuration at reduced crossing angle $\left(\Theta_{c}=450 \mu \mathrm{rad}\right)$, corresponding to the second column of Table I: tune footprint for particles up to $6 \sigma$ betatron amplitude in collision with [right (c) and (d)] or without [left (a) and (b)] LR compensation. 
VV scheme, generally require a substantially larger crossing angle in order to mitigate the LR contribution to the $b_{6}$ like tune spread but also to the bunch to bunch tune variations. In order to run these two schemes at constant (or even further reduced) crossing angle, the LR compensation needs to be activated, assuming as above that the wires operate at a fixed distance with respect to the beam (as calculated for the nominal bunches), but with a modulated current taking into account the Pacman effect. The footprints corresponding to the nominal, PacmanL, and PacmanR bunches, before and after correction, are reported in Figs. 14 and 15 for the $\mathrm{HH}$ and VV configurations, respectively. Before correction, a tune shift of about $\Delta Q_{\mathrm{LR}} \equiv \Delta Q_{x, \mathrm{LR}}=-\Delta Q_{y, \mathrm{LR}} \sim \pm 0.01$ along the antidiagonal is clearly visible for the nominal bunches, which is positive in the $\mathrm{HH}$ configuration, and negative in the $\mathrm{VV}$ configuration [see Figs. 14(b) and 15(b), respectively], in particular pushing the particles toward the third order resonance $3 Q_{y}=1$ in the case of the $\mathrm{VV}$ configuration, and placing the entire footprint below the diagonal in the case of the $\mathrm{HH}$ scheme. This tune shift is reduced by a factor of 2 for the PacmanL and PacmanR bunches, which,

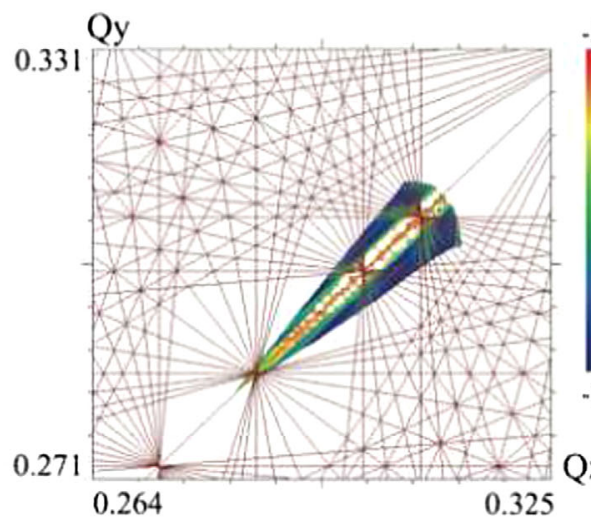

(a)

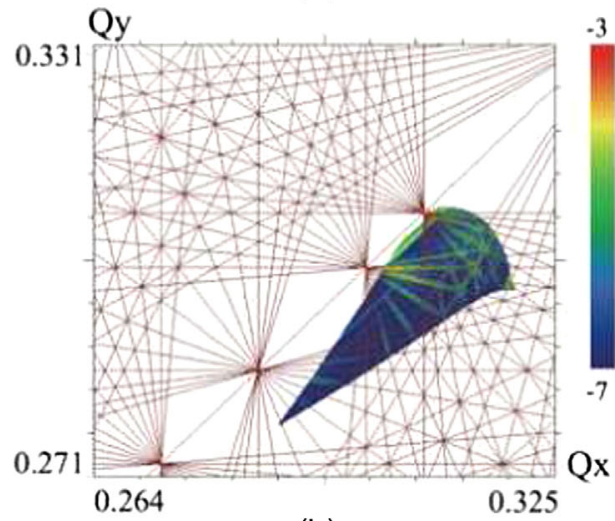

(b)

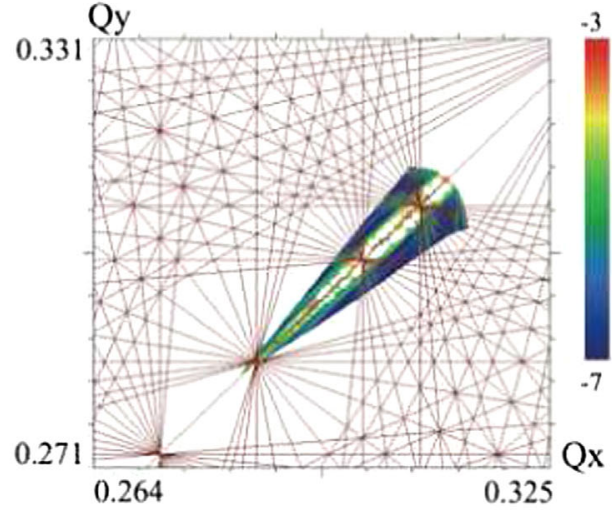

(c)

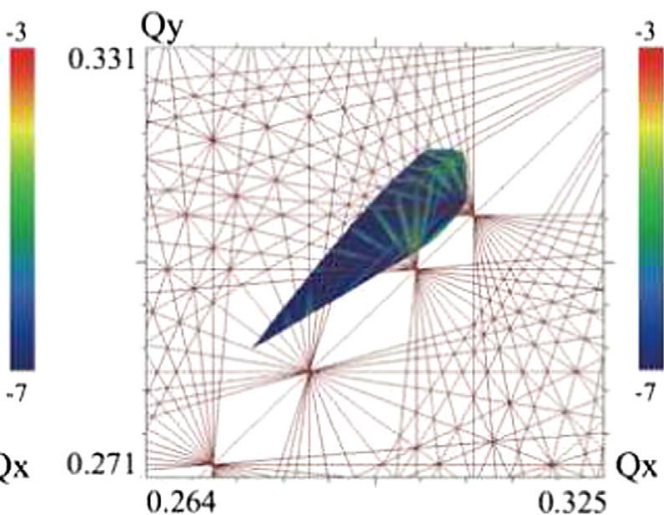

(d)

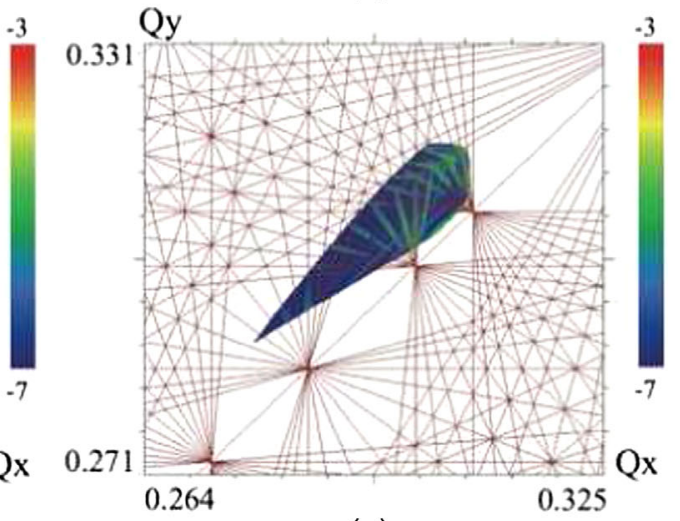

(e)

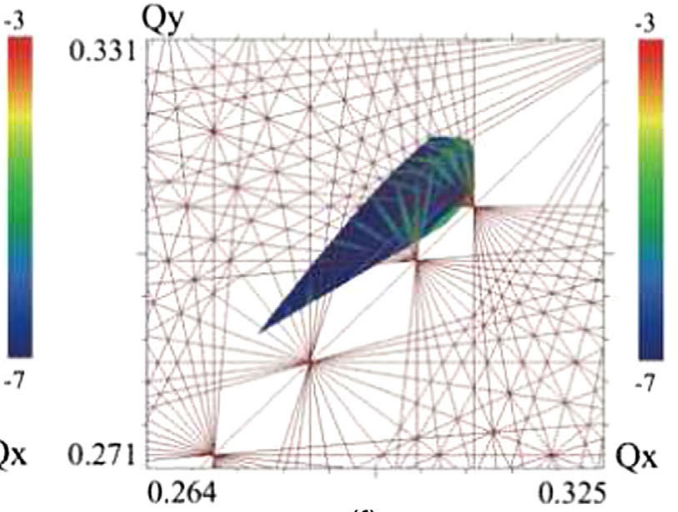

(f)

FIG. 14. PacmanL [(a) and (d)], nominal [(b) and (e)] and PacmanR [(c) and (f)] bunches, HH crossing scheme at nominal crossing angle $\left(\Theta_{c}=590 \mu \mathrm{rad}\right)$, corresponding to the third column of Table I: tune footprint for particles up to $6 \sigma$ betatron amplitude in collision with [right (d), (e) and (f)] or without [left (a), (b) and (c)] LR compensation. 


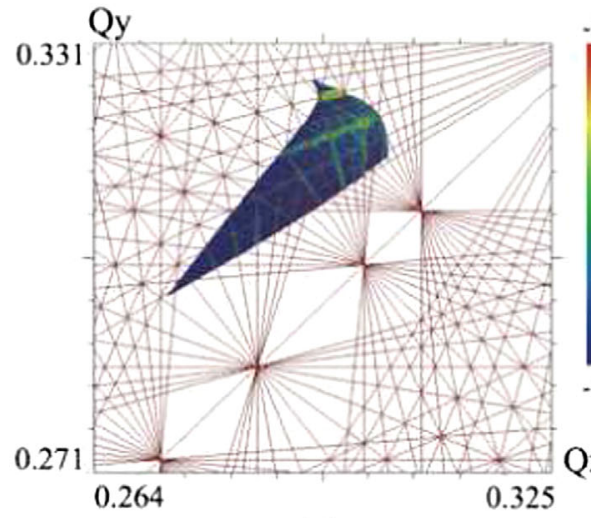

(a)

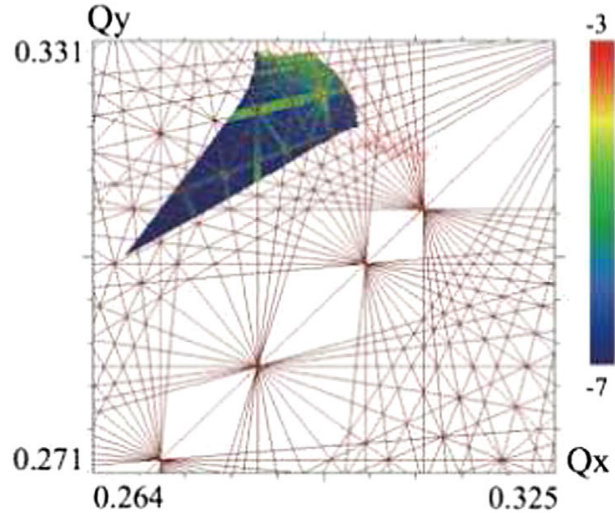

(b)

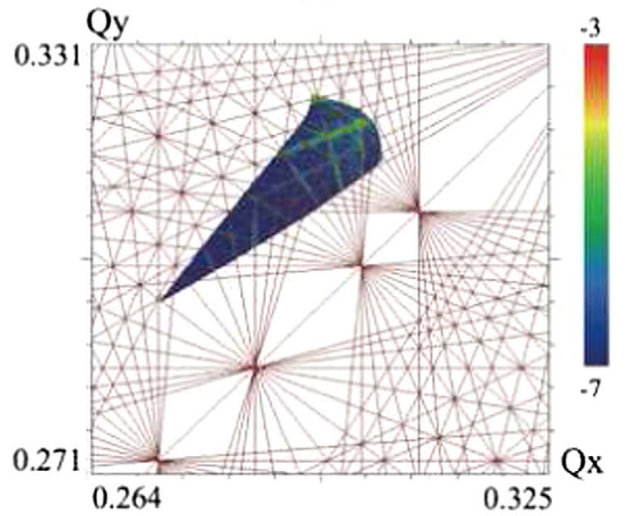

(c)

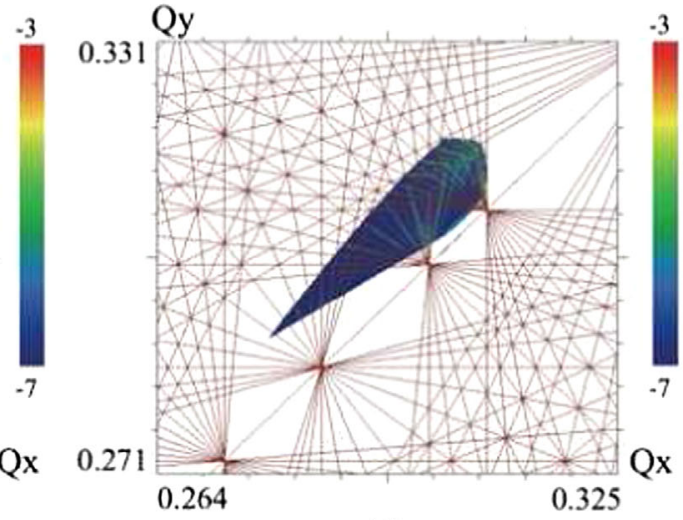

(d)

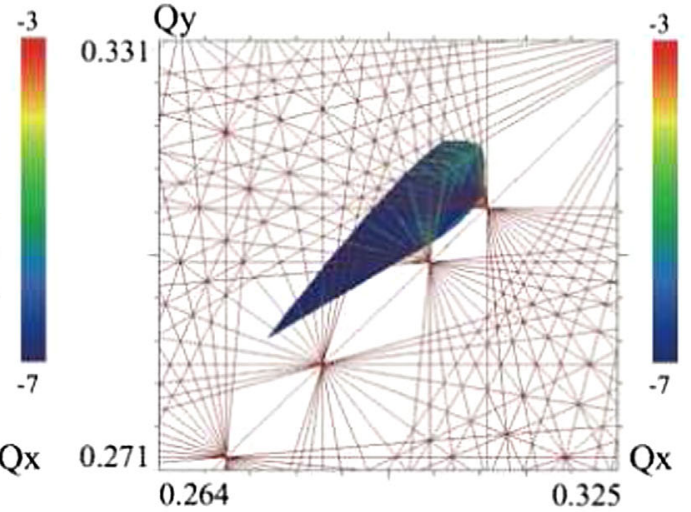

(e)

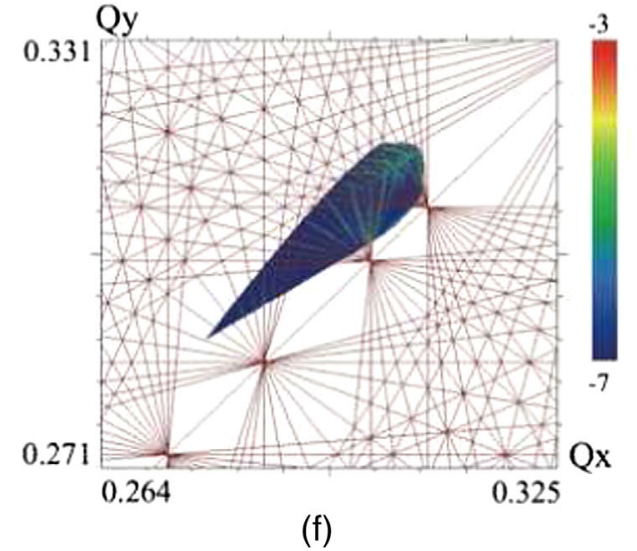

FIG. 15. PacmanL [(a) and (d)], nominal [(b) and (e)] and PacmanR [(c) and (f)] bunches, VV crossing scheme at nominal crossing angle $\left(\Theta_{c}=590 \mu \mathrm{rad}\right)$, corresponding to the fourth column of Table I: tune footprint for particles up to $6 \sigma$ betatron amplitude in collision with [right (d), (e) and (f)] or without [left (a), (b) and (c)] LR compensation.

for the $\mathrm{HH}$ configuration, places the footprint exactly onto the Montague resonance $2 Q_{x}-2 Q_{y}=0$, [see Figs. 14(a) and 14(c)], while improving the situation with respect to the third order resonance in the VV configuration [see Figs. 15(a) and 15(c)]. This variety of cases and subsequent high complexity is then greatly simplified after correction where the footprints are quasi-identical for Pacman and nominal bunches and the distinction between the $\mathrm{HH}$ and VV configurations becomes also rather difficult.
Furthermore, tracking studies not reported here in detail, have shown a dynamic aperture larger than or equal to $8 \sigma$ in each of these cases after wire correction, therefore enabling a reduction of the crossing angle below $590 \mu \mathrm{rad}$ as it was the case for the HV crossing configuration discussed in Sec. III A. These results clearly open up the possibility of an HH or VV crossing scheme for the HL-LHC, with all the advantages that such a configuration may yield as discussed above. 


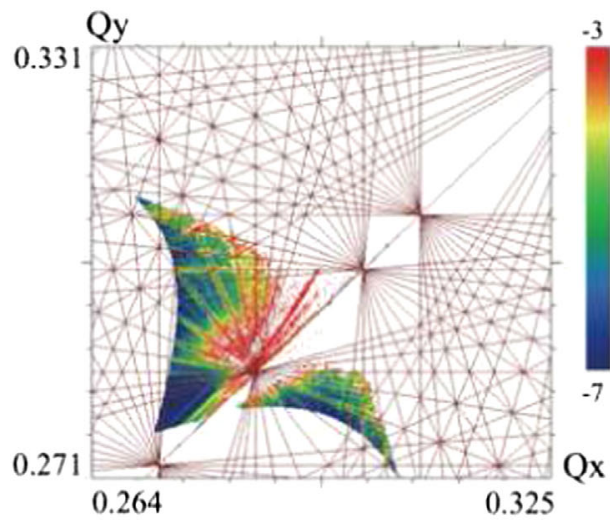

(a)

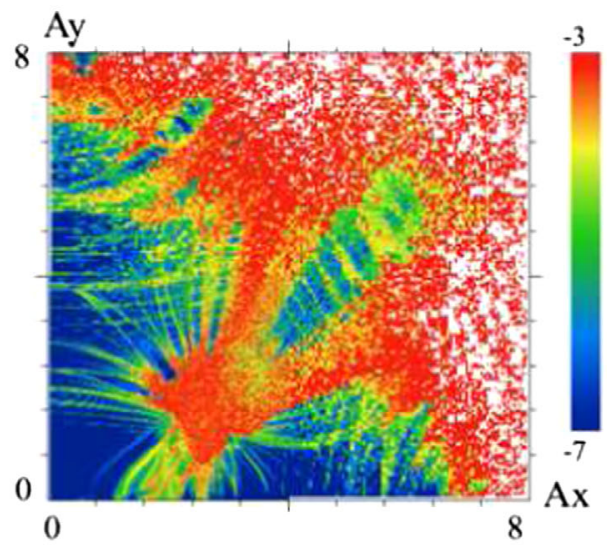

(b)

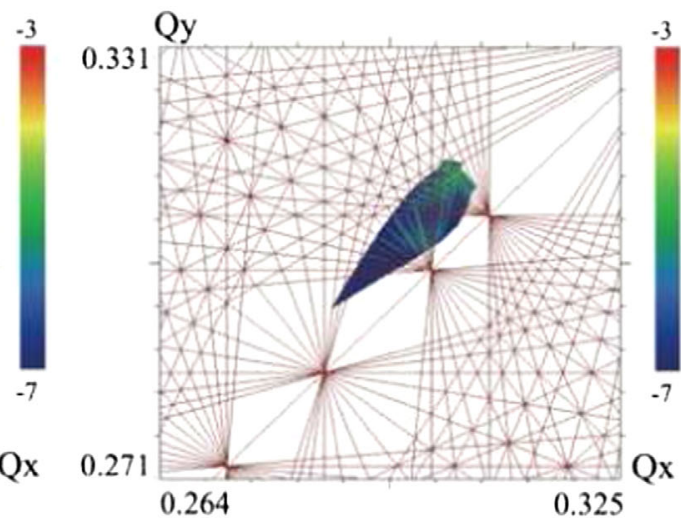

(c)

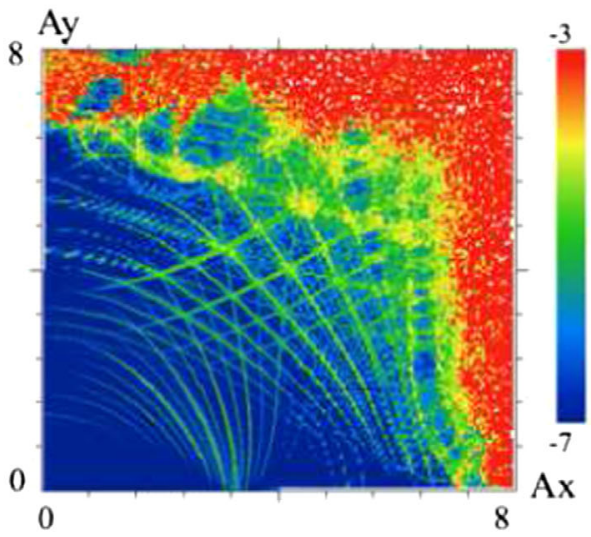

(d)

FIG. 16. Nominal bunches and HL-LHC alternative with flat optics and small crossing angle $\left(\Theta_{c}=300 \mu\right.$ rad $)$, corresponding to the fifth column of Table I: tune footprint for particles up to $6 \sigma$ betatron amplitude [(a) and (c)], and frequency diffusion map for on-momentum particles up to $8 \sigma$ betatron amplitude [(b) and (d)], shown in collision with [(c) and (d)] or without [(a) and (b)] LR compensation.

\section{HL-LHC alternative with flat optics and no crab-cavities}

The last configuration we will discuss is an alternative to the HL-LHC baseline. Without using crab-cavities, this option is very competitive in terms of performance. The configuration relies on flat optics with a substantially smaller crossing angle in the plane of the largest $\beta^{*}$ $\left(\Theta_{c}=300 \mu \mathrm{rad}\right.$, which corresponds to about $10.5 \sigma$ for $\beta^{*}=40 \mathrm{~cm}$ in the crossing plane, see fifth column in Table I). The crossing scheme is alternated similarly to the baseline configuration, vertical in IR1 and horizontal in IR5, with $\beta_{x}^{*} / \beta_{y}^{*} \equiv 10 / 40 \mathrm{~cm}$ at IP1, and conversely at IP5. On the other hand, despite this alternated crossing configuration, the self-compensation of the $(4 n+2)$-pole detuning terms between the two low- $\beta$ insertions is rather limited for flat optics: the overall LR tune shift still corresponds to $75 \%$ of the contribution of one single insertion (assuming a $\beta^{*}$ aspect ratio of 4 and $1 / 4$ in IR1 and IR5 in the present case), and the detuning terms in a given transverse plane (from $b_{4}, b_{6}$, and higher order harmonics) are fully dominated by the contribution of the insertion with the smallest $\beta^{*}$ in the plane considered, without any possibility of compensation by the contribution

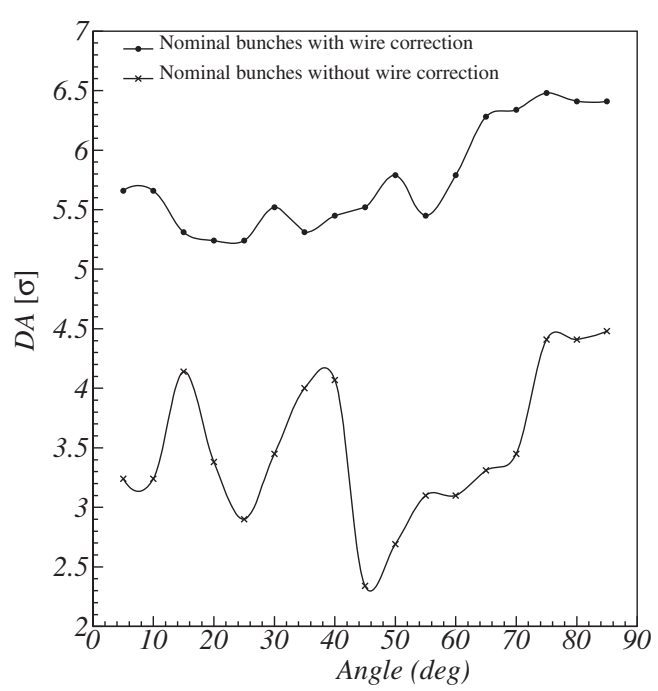

FIG. 17. Nominal bunches and HL-LHC alternative with flat optics and small crossing angle $\left(\Theta_{c}=300 \mu \mathrm{rad}\right)$, corresponding to the fifth column of Table I: 1,000,000 turns dynamic aperture in collision, as a function of the phase space angle, with or without LR compensation, expressed in terms of beam sigma. Each particle is tracked with an initial momentum offset of $2.7 \times 10^{-4}$ corresponding to two thirds of the LHC RF bucket half-height at $7 \mathrm{TeV}$. 
of the other IR. Running the machine under these conditions clearly requires the implementation of a robust LR compensation scheme. As shown in Fig. 16(a), the tune footprint is indeed not only shifted along the diagonal without wire correction $\left(\Delta Q_{x, \mathrm{LR}}=\Delta Q_{y, \mathrm{LR}} \sim-0.015\right.$ for nominal bunches at full intensity), but the LR beam-beam induced tune spread makes it very fat and elongated in the direction of the antidiagonal. The frequency map analysis also clearly shows the excitation of strong resonances at very low amplitude [see Fig. 16(b)] and the DA is even below $2.5 \sigma$ for some directions of the physical space without wire correction [see Fig. 17]. Even under these extreme conditions, however, a sound performance is almost fully recovered after correction with a dynamic aperture of about $5.5 \sigma$ for the nominal bunches at full intensity, an almost head-on like tune footprint can be shaped [see Fig. 16(c)], and most if not all the resonance at low betatron amplitudes have essentially disappeared [see Fig. 16(d)].

\section{SUMMARY AND OUTLOOK}

A generic algorithm has been devised for the compensation of the long-range beam-beam interactions with the use of current bearing wires (or e-lenses) carefully positioned in the layout, where the relevant optimization parameter is the aspect ratio of the $\beta$ functions at the wire position. This analysis revealed in particular an optimal area located in between the matching quadrupoles Q4 and Q5 of the (HL-)LHC where the integration constraints for new equipments are relatively relaxed.

Such a device opens up new possibilities for the HL-LHC in terms of crossing scheme orientations in the two new high-luminosity insertions (HV, HH, VV scheme), in terms of crossing angle reduction, but also in terms of possible HL-LHC overall concept without crab-cavities. All these configurations have been studied in detail with respect to single-particle dynamics related aspects, and the beneficial impact of the correction has been clearly established.

The list of intrinsic advantages or specific features of each of these options has however only been briefly discussed, but it remains extremely relevant. This list indeed contains various aspects which are of very different nature, going from the possibility of optimizing the luminosity lifetime of the new inner triplets via careful crossing angle gymnastics, to a novel HL-LHC concept without crab-cavities but nearly preserved performance (within 10\%), both in terms of data quantity (integrated luminosity) and data quality (line pile-up density). Such a constellation of possibilities gives a very high score to longrange beam-beam compensation devices for the LHC luminosity upgrade, although detailed design and integration studies, and the interface of this device with various accelerator systems, such as the collimation and machine protection systems, remain to be done.

\section{ACKNOWLEDGMENTS}

This research was supported by DOE via the US-LARP program and by EU FP7 HiLumi LHC [34]—Grant Agreement No. 284404.

[1] Edited by O. Brüning, P. Collier, P. Lebrun, S. Myers, R. Ostojic, J. Pool, and P. Proudlock, CERN Report No. CERN-2004-003-V-1, 2004.

[2] L. Rossi, in Proceedings of the 2nd International Particle Accelerator Conference, San Sebastiáán, Spain (EPS-AG, Spain, 2011), p. 908.

[3] S. Fartoukh, in 1st HiLumi WP2 Task leader Meeting, CERN, Geneva, Switzerland, 2012, https://indico.cern.ch/ event/180058/.

[4] S. Fartoukh, CERN Report No. SLHC-Project-Report 49, 2010.

[5] D. Banfi et al., in The Fourth Joint HiLumi LHC-LARP annual meeting, 17-21 November 2014, KEK, Japan, https://indico.cern.ch/event/326148.

[6] J. P. Koutchouk, CERN Report No. LHC-Project-Note 223, 2000.

[7] F. Zimmermann, P. Lebrun, T. Sen, V. Shiltsev, and X. L. Zhang, CERN Report No. CERN AB-Note-2004-041, 2004.

[8] A. Valishev and G. Stancari, Electron lens as beam-beam wire compensator in HL-LHC, arXiv:1312.1660.

[9] S. Fartoukh, in ECFA High Luminosity LHC Experiment Workshop, 2013, Aix-Les-Bains, http://indico.cern.ch/ event $/ 252045 /$.

[10] S. Fartoukh, A. Valishev, and D. Shatilov, in Proceedings of the 5th International Particle Accelerator Conference 2015, May 3-8 2015, Richmond, VA, USA (to be published).

[11] R. B. Palmer, eConf C8806271, 613 (1988); Report No. SLAC-PUB-4707.

[12] R. Calaga, R. Tomas, and F. Zimmermann, CERN Report No. CERN-2007-02, p. 77.

[13] C. Milardi, D. Alesini, M. A. Preger, P. Raimondi, D. Shatilov, and M. Zobov, Report No. CERN-2008-006; Dafne lifetime optimization with compensating wires and octupoles, arXiv:0803.1544.

[14] U. Dorda, J. Koutchouk, R. Tomas, J. Wenninger, F. Zimmermann, R. Calaga, and W. Fischer, in Proceedings of the 11th European Particle Accelerator Conference, Genoa, 2008 (EPS-AG, Genoa, Italy, 2008), p. 3176.

[15] F. Zimmermann, 10 years of wire excitation experiments in the CERN SPS, arXiv:1410.3654.

[16] R. Calaga, W. Fischer, G. Robert-Demolaize, and N. Milas, Long-range beam-beam experiments in the Relativistic Heavy Ion Collider, Phys. Rev. ST Accel. Beams 14, 091001 (2011).

[17] B. Erdelyi and T. Sen, Report No. FERMILAB-TM-2268AD, 2004.

[18] A. Chao and S. Peggs, Report No. SSC-N-80, 1985.

[19] D. Neuffer and S. Peggs, Report No. SSC-63, 1986.

[20] W. Herr, Reports No. CERN SL/93-45 (AP), No. LHCNote-258, 1993.

[21] S. Fartoukh, Report No. CERN-ACC-SLIDES-2014-0113.

[22] X. Buffat, W. Herr, N. Mounet, T. Pieloni, and S. White, Stability diagrams of colliding beams in the Large 
Hadron Collider, Phys. Rev. ST Accel. Beams 17, 111002 (2014).

[23] S. Fartoukh and R. De Maria, in Proceedings of the 3rd International Particle Accelerator Conference, New Orleans, LA, 2012 (IEEE, Piscataway, NJ, 2012), p. 145.

[24] R. de Maria (private communication).

[25] S. Fartoukh, Achromatic telescopic squeezing scheme and its application to the LHC and its luminosity upgrade, Phys. Rev. ST Accel. Beams 16, 111002 (2013).

[26] D. Shatilov, E. Levichev, E. Simonov, and M. Zobov, Application of frequency map analysis to beam-beam effects study in crab waist collision scheme, Phys. Rev. ST Accel. Beams 14, 014001 (2011).

[27] Y. Papaphilippou, Detecting chaos in particle accelerators through the frequency map analysis method, Chaos 24, 024412 (2014).
[28] D. Shatilov, Y. Alexahin, V. Lebedev, and A. Valishev, in Proceedings of the 21st Particle Accelerator Conference, Knoxville, TN, 2005 (IEEE, Piscataway, NJ, 2005), p. 4138.

[29] http://sixtrack.web.cern.ch/SixTrack/.

[30] https://espace.cern.ch/HiLumi/PLC.

[31] S. Fartoukh, Pile up management at the high-luminosity LHC and introduction to the crab-kissing concept, Phys. Rev. ST Accel. Beams 17, 111001 (2014).

[32] S. Fartoukh, M. Ilaria Besana, F. Cerutti, and L. S. Esposito, in 225th LHC Machine Committee, 8 July 2015, https://espace.cern.ch/lhc-machine-committee/.

[33] The LHCb Collaboration, Reports No. CERN-LHCC2013-021, No. LHCB-TDR-013, 2013.

[34] http://hilumilhc.web.cern.ch. 